\title{
POLYNOMIAL TAU-FUNCTIONS OF THE KP, BKP, AND THE $s$-COMPONENT KP HIERARCHIES
}

\author{
VICTOR G. KAC, NATASHA ROZHKOVSKAYA, AND JOHAN VAN DE LEUR
}

\begin{abstract}
We show that any polynomial tau-function of the $s$-component KP and the BKP hierarchies can be interpreted as a zero mode of an appropriate combinatorial generating function. As an application, we obtain explicit formulas for all polynomial tau-functions of these hierarchies in terms of Schur polynomials and $Q$-Schur polynomials respectively. We also obtain formulas for polynomial tau-functions of the reductions of the $s$-component KP hierarchy associated to partitions in $s$ parts.
\end{abstract}

\section{INTRODUCTION}

In [16] M. Sato introduced the KP hierarchy of evolution equations, and his ideas were further developed by the Kyoto school [1] - [5]. In particular, related hierarchies were introduced and studied in these papers, including the BKP and the DKP hierarchy [3], [5], the modified KP-hierarchy [5], the $s$-component KP hierarchy [1]. M. Sato expressed in [16] solutions of the KP hierarchy through tau-functions, and showed that the set of polynomial tau-functions form an infinite Grassmann manifold and contains the Schur polynomials, see [13] (resp. [12]) for an exposition of the theory of tau-functions for the KP (resp. s-component KP) hierarchy. In [18], [19] Y. You proved that polynomial tau-functions of the BKP, DKP and MDKP hierarchies include Schur $Q$-polynomials.

In the recent series of papers [10], [11], [12] the first and the third authors described all polynomial tau-functions of the KP, BKP, DKP, MDKP, s-component KP, and the modified KP hierarchies. In particular, they showed in [10], [12] that any polynomial tau-function of the KP-hierarchy is obtained from a Schur polynomial by certain shifts of arguments, and in [11] they proved that any polynomial tau-function of the BKP or the DKP hierarchy is described by a Pfaffian formula that can be also related to Schur $Q$-polynomials by certain shifts of arguments. In the recent paper [15] the second author showed that the modes of certain generating series, involving Schur Q-polynomials, are tau-functions of the BKP hierarchy.

In this note we show that any polynomial tau-function of the KP, the BKP and the $s$-component KP hierarchy can be interpreted as a zero-mode of an appropriate combinatorial generating function. We give explicit formulae for these generating functions, which have determinant type in the KP and $s$-component KP cases, and Pfaffian type in the BKP case. As a result we recover the formulas for all polynomial tau-functions of the KP and the BKP hierarchies obtained in [10] and [11]. Moreover, we find a new beautiful formula for all polynomial tau-functions of the $s$-component KP hierarchy that generalizes the one for $s=1$ from [10], and its reductions associated to arbitrary partitions in $s$ parts introduced in [9].

The main instrument of our proofs is the identification of the polynomials of the boson space with symmetric functions. Using the boson-fermion correspondence and the well-known properties of symmetric functions, we consider the explicit description of the actions of charged free fermions and neutral fermions on these spaces of symmetric functions. Application of these quantum fields

2010 Mathematics Subject Classification. Primary 17B65, Secondary 35Q53, 20G43, 05E05. 
to the vacuum vector produces the generating functions for polynomial tau-functions, which are identified with solutions of the corresponding bilinear identities.

In Section 2 we review necessary facts on symmetric functions. We describe polynomial taufunctions for the KP case in Section 3, the BKP case in Section 4, and the $s$-component KP case in Section 5 . We treat the polynomial tau-functions of the reductions of the $s$-component KP hierarchy associated to partitions in $s$ parts in Section 6.

Throughout the paper $\mathbb{N}$ (resp. $\mathbb{Z}_{+}$) denotes the set of positive (resp. non-negative) integers.

Acknowledgments. We thank the referee for suggesting some improvements.

\section{Symmetric FUnCTIONS AND FORMAL DISTRIBUTIONS: OVERVIEW}

2.1. Symmetric functions. Recall the definition and properties of symmetric functions [14], [17]. Let $\mathbf{x}=\left\{x_{1}, x_{2}, x_{3} \ldots\right\}$ be an infinite set of indeterminates. Consider the algebra of formal power series $\mathbb{C}[[\mathbf{x}]]=\mathbb{C}\left[\left[x_{1}, x_{2}, x_{3}, \ldots\right]\right]$. By a symmetric function we mean an element of this algebra, invariant with respect to all permutations of the indeterminates, which lies in the span of monomial symmetric functions. Let $\lambda=\left(\lambda_{1} \geq \cdots \geq \lambda_{l} \geq 0\right)$ be a partition. The monomial symmetric function corresponding to $\lambda$ is

$$
m_{\lambda}=\sum_{\left(i_{1}, \ldots, i_{l}\right) \in \mathbb{N}^{l}} x_{i_{1}}^{\lambda_{1}} \ldots x_{i_{l}}^{\lambda_{l}} .
$$

Then the vector space spanned by all monomial symmetric functions is a subalgebra $\Lambda$ of $\mathbb{C}[[\mathbf{x}]]$, called the subalgebra of symmetric functions.

For a partition $\lambda=\left(\lambda_{1} \geq \cdots \geq \lambda_{l} \geq 0\right)$, the Schur symmetric function $s_{\lambda}$ is defined as

$$
s_{\lambda}\left(x_{1}, x_{2}, \ldots\right)=\sum_{T} \mathbf{x}^{T},
$$

where the sum is over all semistandard tableaux of shape $\lambda$ (see e.g. [17] for details). These functions form a basis of the vector space $\Lambda$.

Complete symmetric functions $h_{k}=s_{(k)}$ are given by the formula

$$
h_{k}\left(x_{1}, x_{2} \ldots\right)=\sum_{1 \leq i_{1} \leq \cdots \leq i_{k}<\infty} x_{i_{1}} \ldots x_{i_{k}}
$$

while elementary symmetric functions $e_{k}=s_{\left(1^{k}\right)}$ by

$$
e_{k}\left(x_{1}, x_{2} \ldots\right)=\sum_{1 \leq i_{1}<\cdots<i_{k}<\infty} x_{i_{1}} \ldots x_{i_{k}} .
$$

Power sums $p_{k}$ are symmetric functions defined by

$$
p_{k}\left(x_{1}, x_{2}, \ldots\right)=\sum_{i \in \mathbb{N}} x_{i}^{k} .
$$

It will be convenient to set $h_{-k}\left(x_{1}, x_{2} \ldots\right)=e_{-k}\left(x_{1}, x_{2} \ldots\right)=p_{-k}\left(x_{1}, x_{2} \ldots\right)=0$ for $k>0$ and $h_{0}=e_{0}=p_{0}=1$. Recall that each of these three families generate $\Lambda$ as the algebra of polynomials

$$
\Lambda=\mathbb{C}\left[h_{1}, h_{2}, \ldots\right]=\mathbb{C}\left[e_{1}, e_{2}, \ldots\right]=\mathbb{C}\left[p_{1}, p_{2}, \ldots\right] .
$$

For every symmetric function $f \in \Lambda$ we can associate a polynomial $F\left(t_{1}, t_{2}, \ldots\right)$ by expressing $f$ as a polynomial in the $p_{j}$ 's and replacing $p_{j}$ by $j t_{j}$.

Introduce the polynomials $S_{k}\left(t_{1}, t_{2}, \ldots\right), k \in \mathbb{Z}$, by the generating series

$$
\sum_{k \in \mathbb{Z}} S_{k}\left(t_{1}, t_{2}, \ldots,\right) u^{k}=\exp \left(\sum_{j \in \mathbb{N}} t_{j} u^{j}\right) .
$$


For a partition $\lambda$ having $l$ parts, the corresponding Schur polynomial $S_{\lambda}\left(t_{1}, t_{2}, \ldots\right)$ is defined by the Jacobi-Trudi formula

$$
S_{\lambda}\left(t_{1}, t_{2}, \ldots\right)=\operatorname{det}\left[S_{\lambda_{i}+j-i}\left(t_{1}, t_{2}, \ldots\right)\right]_{1 \leq i, j \leq l} .
$$

This polynomial is associated to the symmetric function $s_{\lambda}$ as described above ([14] I.2 Example 8 and I.3 (3.4)).

We introduce on $\Lambda$ a natural scalar product, where the set of Schur symmetric functions $\left\{s_{\lambda}\right\}$, labeled by partitions $\lambda$, form an orthonormal basis: $\left\langle s_{\lambda}, s_{\mu}\right\rangle=\delta_{\lambda, \mu}$. Then for any linear operator acting on the vector space $\Lambda$ one can define the corresponding adjoint operator. In particular, any symmetric function $f \in \Lambda$ defines an operator of multiplication $f: g \mapsto f g$ for any $g \in \Lambda$, and the corresponding adjoint operator $f^{\perp}$ is defined by the standard rule $\left.<f^{\perp} g_{1}, g_{2}\right\rangle=<g_{1}, f g_{2}>$ for all $g_{1}, g_{2} \in \Lambda$.

It is known ([14], I.5 Example 3) that $p_{n}^{\perp}=n \frac{\partial}{\partial p_{n}}$. Since any element $f \in \Lambda$ can be expressed as a polynomial function of power sums

$$
f=F\left(p_{1}, p_{2}, p_{3}, \ldots\right),
$$

the corresponding adjoint operator $f^{\perp}$ is a polynomial differential operator with constant coefficients

$$
f^{\perp}=F\left(\partial / \partial p_{1}, 2 \partial / \partial p_{2}, 3 \partial / \partial p_{3}, \ldots\right) .
$$

In particular, $e_{k}$ and $h_{k}$ are homogeneous polynomials of degree $k$ in $\left(p_{1}, p_{2}, p_{3}, \ldots\right)$, so the adjoint operators $e_{k}^{\perp}$ and $h_{k}^{\perp}$ are homogeneous polynomials of degree $k$ in $\left(\partial / \partial p_{1}, 2 \partial / \partial p_{2}, \ldots\right)$, which implies the following statement.

Lemma 2.1. For any symmetric function $f \in \Lambda$ there exists a positive integer $N=N(f)$, such that

$$
e_{l}^{\perp}(f)=0 \quad \text { and } \quad h_{l}^{\perp}(f)=0 \quad \text { for all } \quad l \geq N .
$$

2.2. Digression on formal distributions and quantum fields [8]. Given a vector space $M$, an $M$-valued formal distribution is a biletaral series in the indeterminate $u$ with coefficients in $M$ :

$$
a(u)=\sum_{n \in \mathbb{Z}} a_{n} u^{n}, \quad a_{n} \in M .
$$

We denote as $M\left[\left[u, u^{-1}\right]\right]$ the vector space of all $M$-valued formal distributions. We also use the notation $M[u]$ for the space of polynomials, $M[[u]]$ for the space of power series, $M\left[u, u^{-1}\right]$ for the space of Laurent polynomials, and $M((u))$ for the space of formal Laurent series.

A formal distribution in two indeterminates $u$ and $v$ is defined similarly. The most famous example is the formal delta-function $\delta(u, v)$, which is the $\mathbb{C}$-valued formal distribution in variables $u$ and $v$, characterized by the condition

$$
\operatorname{Res}_{u} a(u) \delta(u, v)=a(v)
$$

for any $M$-valued formal distribution $a(u)$, where $\operatorname{Res}_{u}$ denotes the coefficient of $u^{-1}$. The following is an explicit formula:

$$
\delta(u, v)=\sum_{\substack{i, j \in \mathbb{Z} \\ i+j=-1}} u^{i} v^{j}=i_{u, v}\left(\frac{1}{u-v}\right)-i_{v, u}\left(\frac{1}{u-v}\right),
$$

where $i_{u, v}$ (resp. $i_{v, u}$ ) denotes the expansion in geometric series in the domain $|u|>|v|$ (resp. $|u|>$ $|v|)$. The main property of the formal delta-function is that for any $M$-valued formal distribution $a(u)$ one has

$$
a(u) \delta(u, v)=a(v) \delta(u, v) .
$$


A special case of a formal distribution is a quantum field, which is an End $M$-valued formal distribution $a(u)$, such that $a(u) m \in M((u))$ for all $m \in M$. The following lemma is obvious.

Lemma 2.2. If $a(u) \in($ End $M)[[u]]$, and $b(u)$ is a quantum filed, then $a(u) b(u)$ is a quantum field.

2.3. Generating series of polynomial differential operators acting on $\Lambda$. Denote by $\mathcal{D}$ the algebra of differential operators acting on $\Lambda=\mathbb{C}\left[p_{1}, p_{2}, \ldots\right]$, which consists of finite sums

$$
\sum_{i_{1}, \ldots, i_{m}} F_{i_{1}, \ldots i_{m}}\left(p_{1}, p_{2}, \ldots\right) \partial_{p_{1}}^{i_{1}} \ldots \partial_{p_{m}}^{i_{m}}
$$

where coefficients $F_{i_{1}, \ldots i_{m}}\left(p_{1}, p_{2}, \ldots\right)$ are polynomials in $\left(p_{1}, p_{2}, \ldots\right)$. Then operators of multiplication $p_{n}, h_{n}, e_{n}$, their adjoints $p_{n}^{\perp}, h_{n}^{\perp}, e_{n}^{\perp}$ along with their products are elements of $\mathcal{D}$.

Consider the generating series of complete and elementary symmetric functions

$$
H(u)=\sum_{k \in \mathbb{Z}_{+}} h_{k} u^{k}=\prod_{i \in \mathbb{N}} \frac{1}{1-x_{i} u}, \quad E(u)=\sum_{k \in \mathbb{Z}_{+}} e_{k} u^{k}=\prod_{i \in \mathbb{N}}\left(1+x_{i} u\right),
$$

which are elements of $\Lambda[[u]]$. We will use the same notation for the generating series of the corresponding multiplication operators $H(u), E(u) \in \mathcal{D}[[u]]$. Similarly, we define $E^{\perp}(u), H^{\perp}(u) \in$ $\mathcal{D}\left[\left[u^{-1}\right]\right]$ as

$$
E^{\perp}(u)=\sum_{k \in \mathbb{Z}_{+}} \frac{e_{k}^{\perp}}{u^{k}}, \quad H^{\perp}(u)=\sum_{k \in \mathbb{Z}_{+}} \frac{h_{k}^{\perp}}{u^{k}}
$$

The following properties of these generating series with coefficients in $\mathcal{D}$ are well known (e.g. [14], I.5).

Proposition 2.1. We have in $\mathcal{D}[[u]]$ (resp. in $\mathcal{D}\left[\left[u^{-1}\right]\right]$ )

$$
\begin{gathered}
H(u) E(-u)=1, \quad H^{\perp}(u) E^{\perp}(-u)=1, \\
H(u)=\exp \left(\sum_{n \in \mathbb{N}} \frac{p_{n}}{n} u^{n}\right), \quad E(u)=\exp \left(-\sum_{n \in \mathbb{N}} \frac{(-1)^{n} p_{n}}{n} u^{n}\right), \\
E^{\perp}(u)=\exp \left(-\sum_{k \in \mathbb{N}}(-1)^{k} \frac{\partial}{\partial p_{k}} \frac{1}{u^{k}}\right), \quad H^{\perp}(u)=\exp \left(\sum_{k \in \mathbb{N}} \frac{\partial}{\partial p_{k}} \frac{1}{u^{k}}\right),
\end{gathered}
$$

The next set of commutation relations follows from the definitions of the generating series above ([14], I.5 Example 29). These are the main identities that we use to prove other commutation relations later in this note.

Lemma 2.3. We have the following equations in $\mathcal{D}\left[\left[u^{-1}, v\right]\right]$.

$$
\begin{aligned}
& \left(1-\frac{v}{u}\right) E^{\perp}(u) E(v)=E(v) E^{\perp}(u), \\
& \left(1-\frac{v}{u}\right) H^{\perp}(u) H(v)=H(v) H^{\perp}(u), \\
& H^{\perp}(u) E(v)=\left(1+\frac{v}{u}\right) E(v) H^{\perp}(u), \\
& E^{\perp}(u) H(v)=\left(1+\frac{v}{u}\right) H(v) E^{\perp}(u) .
\end{aligned}
$$


2.4. Schur symmetric $Q$-functions and Schur $Q$-polynomials. Introduce one more family of symmetric functions $\left\{q_{k}\left(x_{1}, x_{2}, \ldots\right)\right\}_{k \in \mathbb{Z}}$ as the coefficients of the expansion of $Q(u) \in \Lambda[[u]]$, where

$$
Q(u)=\sum_{k \in \mathbb{Z}} q_{k} u^{k}=E(u) H(u) .
$$

Note that $q_{k}=\sum_{i=0}^{k} e_{i} h_{k-i}$ for $k>0, q_{0}=1$, and $q_{k}=0$ for $q<0$. For $a, b \in \mathbb{Z}_{+}$let

$$
q_{a, b}=q_{a} q_{b}+2 \sum_{i \in \mathbb{Z}}(-1)^{i} q_{a+i} q_{b-i} .
$$

Then ([14], III.8)

$$
q_{a, b}=-q_{b, a} .
$$

Proposition 2.2. ([14], III.8) We have in $\mathcal{D}[[u]]$ (resp. in $\mathcal{D}\left[\left[u^{-1}\right]\right]$ )

$$
\begin{gathered}
Q(u)=S(u)^{2}, \quad \text { where } S(u)=\exp \left(\sum_{n \in \mathbb{N}_{\text {odd }}} \frac{p_{n}}{n} u^{n}\right), \\
S^{\perp}(u)=\exp \left(\sum_{n \in \mathbb{N}_{\text {odd }}} \frac{\partial}{\partial p_{n}} \frac{1}{u^{n}}\right) .
\end{gathered}
$$

Here $\mathbb{N}_{\text {odd }}=\{1,3,5, \ldots\}$.

Let $Q_{k}\left(t_{1}, t_{3}, \ldots\right)$ be the polynomial associated to the symmetric function $q_{k}$ as described above. By (2.9) we have:

$$
Q_{k}\left(t_{1}, t_{3}, \ldots\right)=S_{k}\left(2 t_{1}, 0,2 t_{3}, 0, \ldots\right) .
$$

Recall that the Pfaffian of a skew-symmetric matrix $M=\left[M_{i j}\right]$ of size $2 l \times 2 l$ is defined as

$$
\operatorname{Pf}[M]=\sum_{\sigma \in S_{2 l}^{\prime}} \operatorname{sgn}(\sigma) M_{\sigma(1) \sigma(2)} \cdots M_{\sigma(2 l-1) \sigma(2 l)},
$$

where $S_{2 l}^{\prime}$ is the subset of the permutation group $S_{2 l}$ that consists of $\sigma \in S_{2 l}$ such that $\sigma(2 k-1)<$ $\sigma(2 k)$ for $1 \leq k \leq l$ and $\sigma(2 k-1)<\sigma(2 k+1)$ for $1 \leq k \leq l-1$.

If $\lambda=\left(\lambda_{1}, \ldots, \lambda_{2 m}\right)$ is a strict partition, i.e. $\lambda_{1}>\cdots>\lambda_{2 m} \geq 0$, then the matrix $M_{\lambda}=\left(q_{\lambda_{i}, \lambda_{j}}\right)$ is skew-symmetric by (2.8), and the Schur symmetric $Q$-function $q_{\lambda}$ is defined as

$$
q_{\lambda}\left(x_{1}, x_{2}, \ldots\right)=\operatorname{Pf} M_{\lambda} .
$$

For the strict partition $\lambda=\left(\lambda_{1}, \ldots, \lambda_{2 m}\right)$ introduce the extended $S$ chur $Q$-polynomial $\tilde{Q}_{\lambda}(t)$ as follows:

$$
\begin{aligned}
\tilde{Q}_{a}(t) & =S_{a}(2 t) \quad \text { for } \quad a \in \mathbb{Z}_{+}, \\
\tilde{Q}_{a, b}(t) & =\tilde{Q}_{a}(t) \tilde{Q}_{b}(t)+2 \sum_{i \in \mathbb{Z}}(-1)^{i} \tilde{Q}_{a+i}(t) \tilde{Q}_{a-i}(t) \quad \text { for } \quad a>b, \quad a, b \in \mathbb{Z}_{+}, \\
\tilde{Q}_{a, b}(t) & =-\tilde{Q}_{b, a}(t) \quad \text { for } \quad a<b, \quad a, b \in \mathbb{Z}_{+}, \quad \text { and } \quad Q_{a, a}(t)=0 \quad \text { for } \quad a \in \mathbb{Z}_{+}, \\
\tilde{Q}_{\lambda}(t) & =\operatorname{Pf}\left[\tilde{Q}_{\lambda_{i}, \lambda_{j}}(t)\right] .
\end{aligned}
$$

Then $Q_{\lambda}\left(t_{1}, t_{3}, \ldots\right)=\tilde{Q}_{\lambda}\left(t_{1}, 0, t_{3}, 0 \ldots\right)$ is the Schur $Q$-polynomial associated to the Schur symmetric $Q$-function $q_{\lambda}$. . 
Remark 2.1. One can define a Schur $Q$-polynomial also for a strict partition with odd number of parts, for $\lambda=\left(\lambda_{1}, \lambda_{2}, \ldots, \lambda_{2 m+1}\right)$, define (see [6]):

$$
Q_{\lambda}=Q_{\lambda_{1}} Q_{\lambda_{2}, \lambda_{3}, \ldots, \lambda_{2 m+1}}-Q_{\lambda_{2}} Q_{\lambda_{1}, \lambda_{3}, \ldots, \lambda_{2 m+1}}+\ldots+Q_{\lambda_{2 m+1}} Q_{\lambda_{1}, \lambda_{2}, \ldots, \lambda_{2 m}} .
$$

\section{Polynomial tau-functions of the KP hierarchy}

The goal of this section is to describe polynomial tau-functions of the KP hierarchy. We use the bosonic formulation of the bilinear KP identity and write the action of the Clifford algebra of charged free fermions on the boson Fock space through symmetric functions. Using commutation relations of Lemma 2.3 we prove that all polynomial tau-functions of the KP hierarchy can be obtained as coefficients of certain generating functions. For more information on formal distributions, quantum fields, Clifford algebra of fermions, and the boson-fermion correspondence we refer to [8], [13].

3.1. Fermionic fields on the boson Fock space. Let $\Lambda=\mathbb{C}\left[p_{1}, p_{2}, \ldots\right]$, and consider the boson Fock space $\mathcal{B}=\mathbb{C}\left[z, z^{-1}\right] \otimes \Lambda$. Note that we have the charge decomposition

$$
\mathcal{B}=\oplus_{m \in \mathbb{Z}} \mathcal{B}^{(m)}, \quad \text { where } \quad \mathcal{B}^{(m)}=z^{m} \Lambda .
$$

Let $R(u)$ act on the elements of the form $z^{m} f, f \in \Lambda, m \in \mathbb{Z}$, by the rule

$$
R(u)\left(z^{m} f\right)=z^{m+1} u^{m+1} f,
$$

then $R^{-1}(u)$ acts as

$$
R^{-1}(u)\left(z^{m} f\right)=z^{m-1} u^{-m} f .
$$

Define the formal distributions $\psi^{ \pm}(u)$ of operators acting on the space $\mathcal{B}$ through the action of $R^{ \pm 1}(u)$ and the $\mathcal{D}$-valued generating series $(2.2),(2.3)$ :

$$
\begin{aligned}
& \psi^{+}(u)=u^{-1} R(u) H(u) E^{\perp}(-u), \\
& \psi^{-}(u)=R^{-1}(u) E(-u) H^{\perp}(u),
\end{aligned}
$$

or, in other words, for any $m \in \mathbb{Z}$ and any $f \in \Lambda$,

$$
\begin{aligned}
& \psi^{+}(u)\left(z^{m} f\right)=z^{m+1} u^{m} H(u) E^{\perp}(-u)(f), \\
& \psi^{-}(u)\left(z^{m} f\right)=z^{m-1} u^{-m} E(-u) H^{\perp}(u)(f) .
\end{aligned}
$$

Let the operators $\left\{\psi_{i}^{ \pm}\right\}_{i \in \mathbb{Z}+1 / 2}$ be the coefficients of the expansions

$$
\psi^{ \pm}(u)=\sum_{i \in \mathbb{Z}+1 / 2} \psi_{i}^{ \pm} u^{-i-1 / 2}
$$

Obviously, the operators $\psi_{i}^{ \pm}$change the charge by \pm 1 . These operators are called the charged free fermions.

Proposition 3.1. $\quad$ a) Formulae (3.3) and (3.4) define quantum fields $\psi^{ \pm}(u)$ of operators acting on the space $\mathcal{B}$.

b) The following relations of quantum fields hold:

$$
\begin{aligned}
& \psi^{ \pm}(u) \psi^{ \pm}(v)+\psi^{ \pm}(v) \psi^{ \pm}(u)=0, \\
& \psi^{+}(u) \psi^{-}(v)+\psi^{-}(v) \psi^{+}(u)=\delta(u, v) .
\end{aligned}
$$

Equivalently, (3.3) and (3.4) define the action of the Clifford algebra of charged free fermions on the space $\mathcal{B}$ :

$$
\begin{aligned}
& \psi_{k}^{ \pm} \psi_{l}^{ \pm}+\psi_{l}^{ \pm} \psi_{k}^{ \pm}=0, \\
& \psi_{k}^{+} \psi_{l}^{-}+\psi_{l}^{-} \psi_{k}^{+}=\delta_{k,-l}, \quad k, l \in \mathbb{Z}+1 / 2
\end{aligned}
$$


c) $z \psi_{n}^{ \pm}=\psi_{n \mp 1}^{ \pm} z, \quad n \in \mathbb{Z}+1 / 2$.

Proof. a) Follows from and Lemma 2.1 and Lemma 2.2.

b) By Lemma 2.3, for any $m \in \mathbb{Z}$ and $f \in \Lambda$ we can write

$$
\begin{aligned}
\psi^{+}(u) \psi^{+}(v)\left(z^{m} f\right) & =z^{m+2} u^{m+1} v^{m} H(u) E^{\perp}(-u) H(v) E^{\perp}(-v)(f) \\
& =z^{m+2} u^{m+1} v^{m}\left(1-\frac{v}{u}\right) H(u) H(v) E^{\perp}(-u) E^{\perp}(-v)(f) \\
& =z^{m+2} u^{m} v^{m}(u-v) H(u) H(v) E^{\perp}(-u) E^{\perp}(-v)(f),
\end{aligned}
$$

and, similarly,

$$
\begin{aligned}
\psi^{-}(u) \psi^{-}(v)\left(z^{m} f\right) & =z^{m-2} u^{-m+1} v^{-m} E(-u) H^{\perp}(u) E(-v) H^{\perp}(v)(f) \\
& =z^{m-2} u^{-m+1} v^{-m}\left(1-\frac{v}{u}\right) E(-u) E(-v) H^{\perp}(u) H^{\perp}(v)(f) \\
& =z^{m-2} u^{-m} v^{-m}(u-v) E(-u) E(-v) H^{\perp}(u) H^{\perp}(v)(f) .
\end{aligned}
$$

Switching the roles of $u$ and $v$ and adding the corresponding products of quantum fields we get (3.5).

Using the notation $i_{u, v} F(u, v)$ for the expansion of a rational function $F(u, v)$ in the region $|u|>|v|$, write

$$
i_{u, v}\left(1-\frac{v}{u}\right)^{-1}=1+\frac{v}{u}+\frac{v^{2}}{u^{2}}+\frac{v^{3}}{u^{3}}+\ldots .
$$

It is an inverse of $\left(1-\frac{v}{u}\right)$ in the algebra $\mathcal{D}\left[\left[u^{-1}, v\right]\right]$. Hence we get from Lemma 2.3 that in $\mathcal{D}\left[\left[u^{-1}, v\right]\right]$

$$
E^{\perp}(-u) E(-v)=i_{u, v}\left(1-\frac{v}{u}\right)^{-1} E(-v) E^{\perp}(-u)
$$

and similarly in $\mathcal{D}\left[\left[u, v^{-1}\right]\right]$

$$
H^{\perp}(v) H(u)=i_{v, u}\left(1-\frac{u}{v}\right)^{-1} H(u) H^{\perp}(v) .
$$

For the proof of (3.6), using these relations and claim a), we can write for any $f \in \Lambda$ and any $m \in \mathbb{Z}$,

$$
\begin{gathered}
\psi^{+}(u) \psi^{-}(v)\left(z^{m} f\right)=z^{m} u^{m-1} v^{-m} H(u) E^{\perp}(-u) E(-v) H^{\perp}(v)(f), \\
=i_{u, v}\left(1-\frac{v}{u}\right)^{-1} z^{m} u^{m-1} v^{-m} H(u) E(-v) E^{\perp}(-u) H^{\perp}(v)(f),
\end{gathered}
$$

and similarly,

$$
\psi^{-}(v) \psi^{+}(u)\left(z^{m} f\right)=i_{v, u}\left(1-\frac{u}{v}\right)^{-1} z^{m} u^{m} v^{-m-1} H(u) E(-v) E^{\perp}(-u) H^{\perp}(v)(f) .
$$

Then for the sum of products of quantum fields $\psi^{+}(u) \psi^{-}(v)+\psi^{-}(v) \psi^{+}(u) \in \mathcal{D}\left[\left[u, v, u^{-1}, v^{-1}\right]\right]$ we can write

$$
\begin{aligned}
& \left(\psi^{+}(u) \psi^{-}(v)+\psi^{-}(v) \psi^{+}(u)\right)\left(z^{m} f\right) \\
& =\left(\frac{1}{u} i_{u, v}\left(1-\frac{v}{u}\right)^{-1}+\frac{1}{v} i_{v, u}\left(1-\frac{u}{v}\right)^{-1}\right) \frac{z^{m} u^{m}}{v^{m}} H(u) E(-v) E^{\perp}(-u) H^{\perp}(v)(f) .
\end{aligned}
$$

Note that

$$
\frac{1}{u} i_{u, v}\left(1-\frac{v}{u}\right)^{-1}+\frac{1}{v} i_{v, u}\left(1-\frac{u}{v}\right)^{-1}=i_{u, v}\left(\frac{1}{u-v}\right)+i_{v, u}\left(\frac{1}{v-u}\right)=\delta(u, v) .
$$

Hence

$$
\psi^{+}(u) \psi^{-}(v)+\psi^{-}(v) \psi^{+}(u)\left(z^{m} f\right)=z^{m} \frac{u^{m}}{v^{m}} \delta(u, v) H(u) E(-v) E^{\perp}(-u) H^{\perp}(v)(f)
$$


Using (2.1) and Proposition 2.1 we get

$$
\delta(u, v) \frac{u^{m}}{v^{m}} H(u) E(-v) E^{\perp}(-u) H^{\perp}(v)=\delta(u, v) H(u) E(-u) E^{\perp}(-u) H^{\perp}(u)=\delta(u, v) .
$$

Hence

$$
\left(\psi^{+}(u) \psi^{-}(v)+\psi^{-}(v) \psi^{+}(u)\right)\left(z^{m} f\right)=z^{m} \delta(u, v) f
$$

and (3.6) is proved.

c) is straightforward from definitions.

Remark 3.1. From (2.5), (2.6) one immediately gets the bosonic form of $\psi^{ \pm}(u)$ :

$$
\begin{aligned}
& \psi^{+}(u)=u^{-1} R(u) \exp \left(\sum_{n \geq 1} \frac{p_{n}}{n} u^{n}\right) \exp \left(-\sum_{n \geq 1} \frac{\partial}{\partial p_{n}} \frac{1}{u^{n}}\right), \\
& \psi^{-}(u)=R^{-1}(u) \exp \left(-\sum_{n \geq 1} \frac{p_{n}}{n} u^{n}\right) \exp \left(\sum_{n \geq 1} \frac{\partial}{\partial p_{n}} \frac{1}{u^{n}}\right) .
\end{aligned}
$$

It follows that $\psi_{k-m}^{+}\left(z^{m}\right)=0$ and $\psi_{k+m}^{-}\left(z^{m}\right)=0$ if $k>0$.

3.2. The bilinear KP identity. Recall [2], [3], [13] that the bilinear KP identity is the equation of the form

$$
\Omega(\tau \otimes \tau)=0
$$

on a function $\tau=z^{m} \tau\left(p_{1}, p_{2}, \ldots\right)$ from the formal completion of the space $\mathcal{B}^{(m)}=z^{m} \Lambda$, where

$$
\Omega=\sum_{k \in \mathbb{Z}+1 / 2} \psi_{k}^{+} \otimes \psi_{-k}^{-} .
$$

Note that this equation is independent on $m \in \mathbb{Z}$, By Proposition $3.1 \mathrm{c}) z \tau$ is a solution of the bilinear equation (3.9) iff $\tau$ is. Hence the solutions of (3.9) in $\mathcal{B}^{(m)}$ are obtained from those in $\mathcal{B}^{(n)}$ by multiplying by $z^{m-n}$.

Non-zero solutions of (3.9) are called tau-functions of the KP hierarchy. Accordingly, we will say that a non-zero solution of (3.9) is a polynomial tau-function, if it is a polynomial function in the variables $\left(p_{1}, p_{2}, \ldots\right)$ times $z^{m}$ (hence, an element of $\mathcal{B}^{(m)}$ rather than a completion of the space $\left.\mathcal{B}^{(m)}\right)$. By Remark 3.1, the vector $z^{m}$ is obviously a solution of (3.9). With the help of the commuting with $\Omega$ operators one can construct more examples of tau-functions.

Lemma 3.1. Let $X=\sum_{i>M} A_{i} \psi_{i}^{+}$, where $A_{i} \in \mathbb{C}, M \in \mathbb{Z}$. Then $X^{2}=0$.

Proof. Note that by Proposition 3.1 (a) for any $f \in \Lambda$ and $m \in \mathbb{Z}$ there exists $N$, such that $X\left(z^{m} f\right)=\sum_{i=M+1 / 2}^{N+1 / 2} A_{i} \psi_{i}^{+}\left(z^{m} f\right)$, hence $X$ and $X^{2}$ are well-defined operators on $\mathcal{B}$. Commutation relations (3.7) complete the proof.

Lemma 3.2. Let $X=\sum_{i>M} A_{i} \psi_{i}^{+}$, where $A_{i} \in \mathbb{C}, M \in \mathbb{Z}$. Then

$$
\Omega(X \otimes X)=(X \otimes X) \Omega .
$$


Proof. Note that $\psi_{-k}^{-} X=-X \psi_{-k}^{-}+A_{k}$. Then

$$
\begin{aligned}
\Omega(X \otimes X) & =\sum_{k \in \mathbb{Z}+1 / 2} \psi_{k}^{+} X \otimes \psi_{-k}^{-} X=\sum_{k \in \mathbb{Z}+1 / 2}\left(-X \psi_{k}^{+}\right) \otimes\left(-X \psi_{-k}^{-}+A_{k}\right) \\
& =(X \otimes X) \Omega-X \sum_{k \in \mathbb{Z}+1 / 2} A_{k} \psi_{k}^{+} \otimes 1=(X \otimes X) \Omega-X^{2} \otimes 1=(X \otimes X) \Omega .
\end{aligned}
$$

Corollary 3.1. Let $\tau \in \mathcal{B}^{(m)}$ be a tau-function of the KP hierarchy. Let $X=\sum_{i>M}^{\infty} A_{i} \psi_{i}^{+}$, where $A_{i} \in \mathbb{C}, M \in \mathbb{Z}$. Then $\tau^{\prime}=X \tau \in \mathcal{B}^{(m+1)}$ is also a tau-function of the KP hierarchy.

3.3. Generating function for polynomial tau-functions of the KP hierarchy. Let

$$
G\left(u_{1}, \ldots, u_{l}\right)=\prod_{1 \leq i<j \leq l}\left(u_{j}-u_{i}\right) \prod_{i=1}^{l} H\left(u_{i}\right) .
$$

be a formal power series in $u_{1}, \ldots, u_{l}$ with coefficients in the algebra $\Lambda$. From Lemma 2.3 we have

$$
\begin{aligned}
& \psi^{+}\left(u_{l}\right) \ldots \psi^{+}\left(u_{1}\right)\left(z^{k} f\right)=z^{k+l} u_{l}^{k+l-1} \ldots u_{1}^{k} H\left(u_{l}\right) E^{\perp}\left(-u_{l}\right) \ldots H\left(u_{1}\right) E^{\perp}\left(-u_{1}\right)(f) \\
& =z^{k+l} u_{l}^{k+l-1} \ldots u_{1}^{k} \prod_{1 \leq i<j \leq l}\left(1-\frac{u_{i}}{u_{j}}\right) H\left(u_{l}\right) \ldots H\left(u_{1}\right) E^{\perp}\left(-u_{l}\right) \ldots E^{\perp}\left(-u_{1}\right)(f) .
\end{aligned}
$$

In particular,

$$
\psi^{+}\left(u_{l}\right) \ldots \psi^{+}\left(u_{1}\right)\left(z^{k}\right)=z^{k+l} u_{l}^{k} \ldots u_{1}^{k} G\left(u_{1}, . ., u_{l}\right) .
$$

In [10], [12], polynomial tau-functions of the KP hierarchy are described as determinants of Jacobi-Trudi type. Below we describe polynomial tau-functions as coefficients of certain family of generating functions that have a form similar to (3.10).

Consider a collection of formal Laurent series $A_{1}(u), \ldots, A_{l}(u) \in \mathbb{C}((u))$. Define the $\Lambda$-valued formal Laurent series $T_{i}(u)=A_{i}(u) H(u) \in \Lambda((u)), i=1, \ldots, l$, and let $T_{i ; k} \in \Lambda$ be the coefficients of the expansion

$$
T_{i}(u)=\sum_{k \in \mathbb{Z}} T_{i ; k} u^{k} \in \Lambda((u)), \quad i=1, \ldots, l .
$$

Define also the formal Laurent series in the variables $u_{1}, \ldots, u_{l}$

$$
T\left(u_{1}, \ldots, u_{l}\right)=\prod_{1 \leq i<j \leq l}\left(u_{j}-u_{i}\right) \prod_{i=1}^{l} A_{i}\left(u_{i}\right) H\left(u_{i}\right) \in \Lambda\left[\left[u_{1}, \ldots, u_{l}\right]\right]\left[u_{1}^{-1}, \ldots, u_{l}^{-1}\right] .
$$

For any vector $\alpha=\left(\alpha_{1}, \ldots, \alpha_{l}\right) \in \mathbb{Z}^{l}$ let $T_{\alpha}$ be the coefficient of the corresponding monomial in the expansion

$$
T\left(u_{1}, \ldots, u_{l}\right)=\sum_{\alpha \in \mathbb{Z}^{l}} T_{\alpha} u_{1}^{\alpha_{1}} \ldots u^{\alpha_{l}}
$$

Theorem 3.1. a)

$$
T\left(u_{1}, \ldots, u_{l}\right)=\operatorname{det}\left[u_{i}^{j-1} T_{i}\left(u_{i}\right)\right]_{i, j=1, \ldots, l} .
$$

b) For any $\left(\alpha_{1}, \ldots, \alpha_{l}\right) \in \mathbb{Z}^{l}$ the coefficient $T_{\alpha}$ of the monomial $u_{1}^{\alpha_{1}} \ldots u_{l}^{\alpha_{l}}$ is given by

$$
T_{\alpha}=\operatorname{det}\left[T_{i ; \alpha_{i}+1-j}\right]_{i, j=1, \ldots, l} \text {. }
$$

c) For any $\left(\alpha_{1}, \ldots, \alpha_{l}\right) \in \mathbb{Z}^{l}$ the element $T_{\alpha} \in \Lambda=\mathcal{B}^{(0)}$ is a tau-function of the KP hierarchy. 
d) If $A_{1}(u), \ldots, A_{l}(u) \in \mathbb{C}\left[u, u^{-1}\right]$ are non-zero Laurent polynomials, then the element $T_{\alpha}$ is a polynomial tau-function of the KP hierarchy.

e) Let $\tau \in \mathcal{B}^{(0)}$ be a polynomial tau-function of the KP hiearchy. Then there exists a collection of Laurent polynomials $A_{1}(u), \ldots, A_{l}(u) \in \mathbb{C}\left[u, u^{-1}\right]$ such that $\tau$ is the zero-mode of the Laurent series expansion of (3.12).

Proof. Proof of a) and b) repeats word-to-word calculations of [7] Section 2.2:

$$
\begin{aligned}
T\left(u_{1}, \ldots, u_{l}\right) & =\prod_{i<j}\left(u_{j}-u_{i}\right) \prod_{i=1}^{l} T_{i}\left(u_{i}\right)=\operatorname{det}\left[u_{i}^{j-1}\right] \prod_{i=1}^{l} T_{i}\left(u_{i}\right)=\operatorname{det}\left[u_{i}^{j-1} T_{i}\left(u_{i}\right)\right] \\
& =\sum_{\sigma \in S_{l}} \operatorname{sgn}(\sigma) \sum_{a_{1} \ldots a_{l}} T_{1, a_{1}} u_{1}^{a_{1}+\sigma(1)-1} \cdots T_{l, a_{l}} u_{l}^{a_{l}+\sigma(l)-1} \\
& =\sum_{\alpha_{1} \ldots \alpha_{l}} \sum_{\sigma \in S_{l}} \operatorname{sgn}(\sigma) T_{1, \alpha_{1}+1-\sigma(1)} \cdots T_{l, \alpha_{l}+1-\sigma(l)} u_{1}^{\alpha_{1}} \cdots u_{l}^{\alpha_{l}} \\
& =\sum_{\alpha_{1} \ldots \alpha_{l}} \operatorname{det}\left[T_{i, \alpha_{i}+1-j}\right] u_{1}^{\alpha_{1}} \cdots u_{l}^{\alpha_{l}} .
\end{aligned}
$$

c) From (3.11) we have

$$
A_{l}\left(u_{l}\right) \ldots A_{1}\left(u_{1}\right) \psi^{+}\left(u_{l}\right) \ldots \psi^{+}\left(u_{1}\right)\left(z^{r} \cdot 1\right)=z^{r+l} u_{l}^{r} \ldots u_{1}^{r} T\left(u_{1}, \ldots, u_{l}\right) .
$$

Let $A_{j}(u)=\sum_{k \geq M_{j}} A_{j, k-1 / 2} u^{k}$ (here $\left.A_{j, k-1 / 2} \in \mathbb{C}, M_{j} \in \mathbb{Z}, k \in \mathbb{Z}, j=1, \ldots, l\right)$. Then the coefficient of $u_{1}^{\alpha_{1}} \ldots u_{l}^{\alpha_{l}}$ in $T\left(u_{1}, \ldots, u_{l}\right)$ has the form $T_{\alpha}=z^{-l-r} X_{l} \ldots X_{1}\left(z^{r} \cdot 1\right)$ with

$$
X_{j}=\sum_{i \geq M_{j}-\alpha_{j}+r-1 / 2} A_{j, \alpha_{j}+i-r} \psi_{i}^{+} .
$$

In particular, take $r=0$ to deduce that the coefficient of $u_{1}^{\alpha_{1}} \ldots u_{l}^{\alpha_{l}}$ in $T\left(u_{1}, \ldots, u_{l}\right)$ is a tau-function of the KP hierarchy by Corollary 3.1.

d) If $A_{j, k-1 / 2}=0$ for all $i=1, \ldots, l$ and for all but finitely many $k \in \mathbb{Z}$, the expression $X_{1} \ldots X_{l}(1)$ in c) is a finite linear combination of elements of the form $\psi_{k_{1}}^{+} \ldots \psi_{k_{l}}^{+}(1)$, hence it is a polynomial tau-function.

e) The group $G L_{\infty}$ of automorphisms of the vector space $\mathbb{C}^{\infty}=\oplus_{j \in \mathbb{Z}} \mathbb{C} e_{j}$, which fix all but finitely many $e_{j}$ 's, acts naturally on the semi-infinite wedge space $\bigwedge^{\frac{1}{2}} \mathbb{C}^{\infty}$, for which all semi-infinite monomials of the form $e_{i_{1}} \wedge e_{i_{2}} \wedge e_{i_{3}} \wedge \ldots$, where $i_{1}>i_{2}>i_{3}>\ldots$ and $i_{l+1}=i_{l}-1$ for $l>>0$, form a basis. Let $|m\rangle=e_{m} \wedge e_{m-1} \wedge \ldots$. Through the boson-fermion correspondence the space $\mathcal{B}$ is identified with $\bigwedge^{\frac{1}{2}} \mathbb{C}^{\infty}$, so that $z^{m}$ is identified with $|m\rangle$, see [13] Lecture 5 for more details. Furthermore, the orbit of $G L_{\infty} \cdot 1$ in $\mathcal{B}$ coincides with the set of polynomial tau-functions, see [13] Proposition 7.2.

Let $W$ be the group of permutations of basis vectors $e_{i}$ 's of the vector space $\mathbb{C}^{\infty}$, which fix all but finitely many of them. Let $W_{0} \subset W$ be the subgroup of permutations that fix vectors $e_{i}$ with indices $i>0$. By the Bruhat decomposition of $G L_{\infty}$ (cf. [12]), any element of the orbit of $\mathbb{C}|0\rangle$ has the form $B w(\lambda) \cdot|0\rangle$, where $B=\left(b_{i j}\right)_{i, j \in \mathbb{Z}} \in G L_{\infty}$ is an upper-triangular matrix with 1's on the diagonal $\left(b_{i j}=0\right.$ for $i>j$, and $\left.b_{i i}=1\right)$, and $w(\lambda) \in W / W_{0}$, where $\lambda=\left(\lambda_{1}, \ldots, \lambda_{l}\right)$ is a partition, is defined by:

$$
w(\lambda) \cdot|0\rangle=e_{\lambda_{1}} \wedge \cdots \wedge e_{\lambda_{l}-l+1} \wedge e_{-l} \wedge e_{-l-1} \wedge \ldots
$$


Hence

$$
\begin{aligned}
B w(\lambda) \cdot|0\rangle & =B e_{\lambda_{1}} \wedge \cdots \wedge B e_{\lambda_{l}-l+1} \wedge e_{-l} \wedge e_{-l-1} \wedge \ldots \\
& =f_{1} \wedge f_{2} \wedge \cdots \wedge f_{l} \wedge e_{-l} \wedge e_{-l-1} \wedge \ldots
\end{aligned}
$$

where

$$
f_{j}=\sum_{s=-l+1}^{\lambda_{j}-j+1} b_{s, \lambda_{j}-j+1} e_{s} .
$$

Note that $f_{j}$ is just the 'truncated at the level $-l+1$ ' column $\left(b_{i, \lambda_{j}-j+1}\right)_{i \in \mathbb{Z}}$ of the matrix $B$, due to the operation of wedge product with $|-l\rangle=e_{-l} \wedge e_{-l+1} \wedge \ldots$ Using that $\psi_{-s+1 / 2}^{+}\left(e_{i_{1}} \wedge e_{i_{2}} \ldots\right)=$ $e_{s} \wedge e_{i_{1}} \wedge e_{i_{2}} \ldots$, write

$$
B w(\lambda) \cdot|0\rangle=Y_{1} \ldots Y_{l}|-l\rangle,
$$

where

$$
Y_{j}=\sum_{s=-l+1}^{\lambda_{j}-j+1} b_{s, \lambda_{j}-j+1} \psi_{-s+1 / 2}^{+}, \quad j=1, \ldots, l .
$$

For a fixed vector $\left(\alpha_{1}, \ldots, \alpha_{l}\right) \in \mathbb{Z}^{l}$ set

$$
A_{j}(u)=\sum_{t=\alpha_{j}-\lambda_{j}+j-l}^{\alpha_{j}} A_{j, t-1 / 2} u^{t}, \quad j=1, \ldots, l,
$$

with non-zero terms of the sum defined by entries of the $\lambda_{j}-j+1$-th column of the matrix $B$ :

$$
A_{j, t-1 / 2}=b_{-l+1+\alpha_{j}-t, \lambda_{j}-j+1} .
$$

Then

$$
\sum_{i} A_{j, \alpha_{j}+i+l} \psi_{i}^{+}=\sum_{i} b_{1 / 2-i, \lambda_{j}-j+1} \psi_{i}^{+}=\sum_{s} b_{s, \lambda_{j}-j+1} \psi_{-s+1 / 2}^{+}
$$

and we see that $X_{j}$ in (3.15) coincides with $Y_{j}$ in (3.16). Hence, with such choice of $A_{1}(u), \ldots, A_{l}(u)$, the coefficient $T_{\alpha}$ of $u_{1}^{\alpha_{1}} \ldots u_{l}^{\alpha_{l}}$ in $T\left(u_{1}, \ldots, u_{l}\right)$ coincides with the given polynomial tau-function $Y_{l} \ldots Y_{1}|-l\rangle= \pm B w(\lambda) \cdot|0\rangle$. Note that we proved that for any polynomial tau-function $\tau$ and any given vector $\left(\alpha_{1}, \ldots, \alpha_{l}\right) \in \mathbb{Z}^{l}$ of the appropriate length $l$ there exists $T\left(u_{1}, \ldots, u_{l}\right)$ such that $\tau$ is the coefficient of $u_{1}^{\alpha_{1}} \ldots u_{l}^{\alpha_{l}}$ in $T\left(u_{1}, \ldots, u_{l}\right)$. In particular, we can consider $\alpha_{1}=\cdots=\alpha_{l}=0$ to represent the given polynomial tau-function as the zero-mode of certain $T\left(u_{1}, \ldots, u_{l}\right)$.

\subsection{Remarks and corollaries.}

a) We proved that polynomial tau-functions are zero-modes of appropriate generating functions $T\left(u_{1}, \ldots, u_{l}\right)$, but changing back $A_{j}(u) \mapsto u^{\alpha_{j}} A_{j}(u)$ allows one to get any polynomial taufunction as a coefficient of a given monomial $u_{1}^{\alpha_{1}} \ldots u_{l}^{\alpha_{l}}$.

b) Consider non-zero Laurent series $A_{1}(u), \ldots, A_{l}(u)$ in the definition of $T\left(u_{1}, \ldots, u_{l}\right)$. One can represent each of them in the form

$$
A_{j}(u)=u^{M_{j}} b_{j} \sum_{i=0}^{\infty} a_{j, i} u^{i}
$$


for suitable choice of $M_{j} \in \mathbb{Z}, b_{j}, a_{j, i} \in \mathbb{C}$, and $a_{j, 0}=1, b_{j} \neq 0$. Note that there exists a collection of constants $\left\{c_{j, s}\right\} \subset \mathbb{C}$ such that

$$
\sum_{i=0}^{\infty} a_{j, i} u^{i}=\exp \left(\sum_{s=1}^{\infty} c_{j, s} u^{s}\right)
$$

in other words, $a_{j, i}=S_{i}\left(c_{j, 1}, c_{j, 2}, \ldots\right)$. Then, letting $t_{k}=k p_{k}$, we obtain by $(2.5)$

$$
\begin{aligned}
T_{j}(u) & =A_{j}(u) H(u)=u^{M_{j}} b_{j} \exp \left(\sum_{k=1}^{\infty} c_{j, k} u^{k}\right) \exp \left(\sum_{k=1}^{\infty} t_{k} u^{k}\right) \\
& =u^{M_{j}} b_{j} \exp \left(\sum_{k=1}^{\infty}\left(t_{k}+c_{j, k}\right) u^{k}\right)=u^{M_{j}} b_{j} \sum_{k=0}^{\infty} S_{k}\left(t_{1}+c_{j, 1}, t_{2}+c_{j, 2}, \ldots\right) u^{k} .
\end{aligned}
$$

Hence $T_{j ; k}=b_{j} S_{k-M_{j}}\left(t_{1}+c_{j, 1}, t_{2}+c_{j, 2}, \ldots\right)$, and by Theorem 3.1, polynomial KP taufunctions have the form

$$
T_{\alpha}=\left(\prod_{k} b_{k}\right) \cdot \operatorname{det}\left[S_{\alpha_{i}-M_{i}+1-j}\left(t_{1}+c_{i, 1}, t_{2}+c_{i, 2}, \ldots\right)\right]_{i, j=1, \ldots, l}
$$

for any choice of constants $\left\{c_{j, k}\right\}$. This recovers the description of all polynomial taufunctions of the KP hierarchy of [10] and, in particular, Sato's theorem [16] that all Schur polynomials are tau-functions of the KP hierarchy. Obviously, it suffices to take here for $A_{j}(u)$ Laurent polynomials.

\section{Polynomial tau-functions of the BKP hierarchy}

In this section we describe polynomial tau-functions of the BKP hierarchy as coefficients of certain generating functions.

4.1. Neutral fermions action on the boson Fock space. Through all of Section 4 denote $Q(u)=E(u) H(u)=S(u)^{2}=\sum_{k \in \mathbb{Z}} q_{k} u^{k}$ as in (2.7) and (2.9). Consider the boson Fock space generated by odd power sums:

$$
\mathcal{B}_{\text {odd }}=\mathbb{C}\left[p_{1}, p_{3}, p_{5}, \ldots\right] .
$$

Recall ([14], III.8 (8.5)) that $q_{k} \in \mathcal{B}_{\text {odd }}$, and that $\mathcal{B}_{\text {odd }}=\mathbb{C}\left[q_{1}, q_{3}, \ldots\right]$. From (2.6) it is clear that $\mathcal{B}_{\text {odd }}$ is invariant with respect to action of $e_{k}^{\perp}$, and $h_{k}^{\perp}$, and one can prove [15] that restrictions to $\mathcal{B}_{\text {odd }}$ of the operators $E^{\perp}(u), H^{\perp}(u), S^{\perp}(u)$ coincide. We can define the following formal distribution of operators acting on $\mathcal{B}_{\text {odd }}$ :

$$
\varphi(u)=E(u) H(u) E^{\perp}(-u)=Q(u) S^{\perp}(-u) .
$$

Let $\left\{\varphi_{i}\right\}_{i \in \mathbb{Z}}$ be coefficients of the expansion $\varphi(u)=\sum_{j \in \mathbb{Z}} \varphi_{j} u^{-j}$. Similarly to Proposition 3.1 one proves the following statements [15].

Proposition 4.1. a) Formula (4.1) defines a quantum field $\varphi(u)$ of operators acting on the space $\mathcal{B}_{\text {odd }}$.

b) One has relations

$$
\varphi(u) \varphi(v)+\varphi(v) \varphi(u)=2 v \delta(v,-u),
$$

where $\delta(u, v)$ is the formal delta function. Hence (4.1) is the action of the Clifford algebra of neutral fermions on the space $\mathcal{B}_{\text {odd }}$ :

$$
\varphi_{m} \varphi_{n}+\varphi_{n} \varphi_{m}=2(-1)^{m} \delta_{m+n, 0} \quad \text { for } \quad m, n \in \mathbb{Z} .
$$


Remark 4.1. Using (2.5), (2.6) one gets the bosonic form of $\varphi(u)$ :

$$
\varphi(u)=Q(u) S(-u)^{\perp}=\exp \left(\sum_{n \in \mathbb{N}_{\text {odd }}} \frac{2 p_{n}}{n} u^{n}\right) \exp \left(-\sum_{n \in \mathbb{N}_{\text {odd }}} \frac{\partial}{\partial p_{n}} \frac{1}{u^{n}}\right) .
$$

It follows that $\varphi_{n}(1)=0$ for $n>0$ and $\varphi_{0}(1)=1$.

4.2. The bilinear BKP identity. The bilinear BKP identity [2], [3], [11] is the equation of the form

$$
\Omega(\tau \otimes \tau)=\tau \otimes \tau
$$

on elements $\tau=\tau\left(p_{1}, p_{3}, p_{5} \ldots\right)$ from the completion of $\mathcal{B}_{\text {odd }}$, where

$$
\Omega=\sum_{n \in \mathbb{Z}} \varphi_{n} \otimes(-1)^{n} \varphi_{-n}
$$

Non-zero solutions of (4.3) are called the tau-functions of the BKP hierarchy. We will say that a solution of (4.3) is a polynomial tau-function if it is a polynomial function in the variables $\left(p_{1}, p_{3}, p_{5} \ldots\right)$ (hence, it is an element of $\mathcal{B}_{\text {odd }}$ rather than its completion). By Remark 4.1, $\tau=1$ is a tau-function of the BKP hierarchy. Similarly to Lemma 3.2 and statements of [15] we prove the following lemma.

Lemma 4.1. Let $X=\sum_{n \geq M} A_{n} \varphi_{n}$, where $A_{n} \in \mathbb{C}$ and $M \in \mathbb{Z}$. Then

$$
X^{2}=\sum_{M \leq k \leq-M}(-1)^{k} A_{k} A_{-k} \cdot I d,
$$

in particular, $X^{2}=A_{0}^{2} \cdot$ Id if $M=0$, and $X^{2}=0$ if $M>0$.

Proof. Note that due to Proposition 4.1 part a) $X$ and $X^{2}$ are well-defined operators on the space $\mathcal{B}_{\text {odd }}$. Split $X=X^{-}+A_{0} \varphi_{0}+X^{+}$, where $X^{-}=\sum_{n<0} A_{n} \varphi_{n}, X^{+}=\sum_{n>0} A_{n} \varphi_{n}$. Due to commutation relations (4.2),

$$
\left(X^{ \pm}\right)^{2}=0, \quad \varphi_{0}\left(X^{+}+X^{-}\right)+\left(X^{+}+X^{-}\right) \varphi_{0}=0, \quad \varphi_{0}^{2}=I d,
$$

and

$$
X^{+} X^{-}+X^{-} X^{+}=\sum_{M \leq n<0} 2(-1)^{n} A_{n} A_{-n} \cdot I d=2 \sum_{\substack{M \leq n \leq-M \\ n \neq 0}}(-1)^{n} A_{n} A_{-n} \cdot I d .
$$

if $M<0$, and $X^{+} X^{-}+X^{-} X^{+}=0$ if $M \geq 0$. Applying these identities in the expansion of $X^{2}$ we get

$$
X^{2}=\left(X^{-}+A_{0} \varphi_{0}+X^{+}\right)^{2}=\sum_{k=M}^{-M}(-1)^{k} A_{k} A_{-k} \cdot I d
$$

\section{Lemma 4.2 .}

$$
\Omega(X \otimes X)=(X \otimes X) \Omega .
$$

Proof. For any $n \in \mathbb{Z}$

$$
\varphi_{n} X+X \varphi_{n}=2(-1)^{n} \sum_{k \geq M} A_{k} \delta_{k+n, 0}= \begin{cases}2(-1)^{n} A_{-n}, & \text { if } n \leq-M, \\ 0 & \text { otherwise }\end{cases}
$$


Then

$$
\begin{aligned}
& \Omega(X \otimes X)=(X \otimes X) \Omega-2 \sum_{n \in \mathbb{Z}} \sum_{l \geq M} X \varphi_{n} \otimes A_{l} \delta_{l-n, 0}-2 \sum_{n \in \mathbb{Z}} \sum_{k \geq M} A_{k} \delta_{k+n, 0} \otimes X \varphi_{-n} \\
& +4 \sum_{n \in \mathbb{Z}} \sum_{k, l \geq M}(-1)^{n} A_{k} \delta_{k+n, 0} \otimes A_{l} \delta_{l-n, 0} \\
& =(X \otimes X) \Omega-2\left(X^{2} \otimes 1+1 \otimes X^{2}\right)+\left(4 \sum_{M \leq k \leq-M}(-1)^{k} A_{k} A_{-k}\right) 1 \otimes 1 .
\end{aligned}
$$

Using (4.4) we complete the proof of (4.5).

Corollary 4.1. Let $\tau \in \mathcal{B}_{\text {odd }}$ be a tau-function of the BKP hierarchy, and let $X=\sum_{n \geq M} A_{n} \varphi_{n}$, where $A_{n} \in \mathbb{C}$ and $M \in \mathbb{Z}$. Then $\tau^{\prime}=X \tau$ is also a tau-function of the BKP hierarchy.

\subsection{Generating functions for polynomial tau-functions of the BKP hierarchy. Let}

$$
f(u, v)=i_{u, v}\left(\frac{u-v}{u+v}\right)=\left(1-\frac{v}{u}\right) \sum_{k \in \mathbb{Z}_{+}}(-1)^{k} \frac{v^{k}}{u^{k}}=1+2 \sum_{k \in \mathbb{N}}(-1)^{k} \frac{v^{k}}{u^{k}} \in \mathbb{C}[[v / u]] .
$$

Note that

$$
f(u, v)+f(v, u)=(u-v) \delta(u,-v)=2 \sum_{k \in \mathbb{Z}} \frac{u^{k}}{(-v)^{k}} .
$$

Define a formal distribution $Q\left(u_{1}, \ldots, u_{l}\right)$ with coefficients in $\mathcal{B}_{\text {odd }}$ as a result of application of a product of quantum fields $\varphi\left(u_{i}\right)$ to the vacuum vector $1 \in \mathcal{B}_{\text {odd }}$.

$$
\varphi\left(u_{l}\right) \ldots \varphi\left(u_{1}\right)(1)=Q\left(u_{1}, \ldots, u_{l}\right),
$$

By (4.1) and Lemma 2.3 we get

$$
Q\left(u_{1}, \ldots, u_{l}\right)=\prod_{1 \leq i<j \leq l} f\left(u_{j}, u_{i}\right) \prod_{i=1}^{l} Q\left(u_{i}\right)
$$

where $Q(u)$ is the generating series (2.7). Define the elements $Q_{\alpha} \in \mathcal{B}_{\text {odd }}$ as the coefficients of the formal distribution $Q\left(u_{1}, \ldots, u_{l}\right)=\sum_{\alpha \in \mathbb{Z}^{l}} Q_{\alpha} u_{1}^{\alpha_{1}} \ldots u_{l}^{\alpha_{l}}$. Note that (compare with Theorem 4.28 in [6])

$$
Q_{\alpha}=\varphi_{-\alpha_{1}} \ldots \varphi_{-\alpha_{l}}(1)
$$

Let $A_{1}(u), \ldots, A_{l}(u) \in \mathbb{C}\left[u, u^{-1}\right]$ be a collection of Laurent polynomials. Define a $\mathcal{B}_{\text {odd }}$-valued formal distribution

$$
T\left(u_{1}, \ldots, u_{l}\right)=\prod_{i=1}^{l} A_{i}\left(u_{i}\right) Q\left(u_{1}, \ldots, u_{l}\right)
$$

For any $\alpha=\left(\alpha_{1}, \ldots, \alpha_{l}\right) \in \mathbb{Z}^{l}$ let $T_{\alpha}$ be the coefficient in the expansion

$$
T\left(u_{1}, \ldots, u_{l}\right)=\sum_{\alpha \in \mathbb{Z}^{l}} T_{\alpha} u_{1}^{\alpha_{1}} \ldots u_{l}^{\alpha_{l}}
$$


Remark 4.2. Note that for any choice of Laurent polynomials $A_{1}(u), \ldots, A_{2 l-1}(u)$ and with $A_{2 l}(u)=$ 1 in (4.8) we have

$$
T\left(u_{1}, u_{2}, \ldots, u_{2 l-1}\right)=-T\left(u_{1}, u_{2}, \ldots, u_{2 l-1}, 0\right) .
$$

Hence, it is sufficient to consider only the case of even number of variables $\left(u_{1}, \ldots, u_{2 l}\right)$.

Let $\left(u_{1}, u_{2}, \ldots, u_{l}\right)$ be a collection of variables. Introduce the matrix $\tilde{F}=\left[\tilde{f}_{i, j}\right]_{i, j=1, \ldots, 2 l}$ with coefficients

$$
\tilde{f}_{i, j}=\left\{\begin{array}{cc}
f\left(u_{i}, u_{j}\right), & \text { if } \quad i<j \\
0, & \text { if } i=j \\
-f\left(u_{j}, u_{i}\right), & \text { if } \quad i>j
\end{array}\right.
$$

For $i, j \in\{1, \ldots, 2 l\}$ consider formal distributions

$$
T^{(i)}\left(u_{i}\right)=A_{i}\left(u_{i}\right) Q\left(u_{i}\right) \quad \text { and } \quad \tilde{T}^{(i, j)}\left(u_{i}, u_{j}\right)=\tilde{f}_{i, j} T^{(i)}\left(u_{i}\right) T^{(j)}\left(u_{j}\right) .
$$

Note that $\tilde{T}^{(i, j)}\left(u_{i}, u_{j}\right)=-\tilde{T}^{(j, i)}\left(u_{j}, u_{i}\right)$.

For any $(a, b) \in \mathbb{Z}^{2}$ and $i<j$ let $\tilde{T}_{a, b}^{(i, j)}$ be the coefficients of the expansion

$$
\tilde{T}^{(i, j)}\left(u_{i}, u_{j}\right)=\sum_{a, b \in \mathbb{Z}} \tilde{T}_{a, b}^{(i, j)} u_{i}^{a} u_{j}^{b}
$$

For $i>j$ set $\tilde{T}_{a, b}^{(i, j)}=-\tilde{T}_{a, b}^{(j, i)}$, and set $\tilde{T}_{a, b}^{(i, i)}=0$.

Theorem 4.1. a)

$$
T\left(u_{1}, u_{2}, \ldots, u_{2 l}\right)=\operatorname{Pf}\left[\tilde{T}^{(i, j)}\left(u_{i}, u_{j}\right)\right]_{i, j=1, \ldots, 2 l} .
$$

b) For any $\left(\alpha_{1}, \ldots, \alpha_{2 l}\right) \in \mathbb{Z}^{2 l}$ (see Remark 4.2 for the odd case) the coefficient $T_{\alpha}$ of the monomial $u_{1}^{\alpha_{1}} \ldots u_{2 l}^{\alpha_{2 l}}$ in (4.9) equals

$$
T_{\alpha}=\operatorname{Pf}\left[\tilde{T}_{\alpha_{i}, \alpha_{j}}^{(i, j)}\right]_{i, j=1, \ldots, 2 l} .
$$

c) For any $\left(\alpha_{1}, \ldots, \alpha_{l}\right) \in \mathbb{Z}^{l}$ the coefficient $T_{\alpha}$ of $u_{1}^{\alpha_{1}} \ldots u_{l}^{\alpha_{l}}$ in (4.9) is a polynomial taufunction of the BKP hierarchy.

d) Let $\tau$ be a polynomial tau-function of the BKP hierarchy. Then there exists a collection of Laurent polynomials $A_{1}(u), \ldots, A_{l}(u)$ such that $\tau$ is the zero-mode of the series expansion of (4.8).

Proof. The proof of of a) and b) follows the steps of the proof of similar statement in [7], Section 2.3. Recall ([14], III.8) the equality of rational functions

$$
\operatorname{Pf}\left[\frac{u_{i}-u_{j}}{u_{i}+u_{j}}\right]_{i, j=1, \ldots, 2 l}=\prod_{1 \leq i<j \leq 2 l} \frac{u_{i}-u_{j}}{u_{i}+u_{j}} .
$$

Let $i\left(g\left(u_{1}, \ldots, u_{l}\right)\right)$ be the series expansion of the rational function $g\left(u_{1}, \ldots, u_{l}\right)$ in the variables $u_{1}, \ldots, u_{l}$ in the region $\left|u_{1}\right|>\cdots>\left|u_{l}\right|$. Then $i\left(\frac{u_{i}-u_{j}}{u_{i}+u_{j}}\right)=\tilde{f}\left(u_{i}, u_{j}\right)$, and

$$
i\left(\operatorname{Pf}\left[\frac{u_{i}-u_{j}}{u_{i}+u_{j}}\right]\right)=\sum_{\sigma \in S_{2 l}^{\prime}} \operatorname{sgn}(\sigma) \tilde{f}_{\sigma(1) \sigma(2)} \ldots \tilde{f}_{\sigma(2 l-1) \sigma(2 l)}=\operatorname{Pf} \tilde{F} .
$$

Expanding the rational function in the right-hand side of (4.10) we get

$$
\operatorname{Pf} \tilde{F}=\prod_{1 \leq i<j \leq 2 l} f\left(u_{i}, u_{j}\right)
$$


Then

$$
\begin{aligned}
T\left(u_{1}, u_{2}, \ldots, u_{2 l}\right) & =\prod_{1 \leq i<j \leq 2 l} f\left(u_{i}, u_{j}\right) \prod_{i=1}^{2 l} T^{(i)}\left(u_{i}\right)=\operatorname{Pf} \tilde{F} \prod_{i=1}^{2 l} T^{(i)}\left(u_{i}\right) \\
& =\sum_{\sigma \in S_{2 l}^{\prime}} \operatorname{sgn}(\sigma) \tilde{f}_{\sigma(1) \sigma(2)} \cdots \tilde{f}_{\sigma(2 l-1) \sigma(2 l)} \prod_{i=1}^{2 l} T^{(i)}\left(u_{i}\right) \\
& =\sum_{\sigma \in S_{2 l}^{\prime}} \operatorname{sgn}(\sigma) \tilde{f}_{\sigma(1) \sigma(2)} T^{\sigma(1)}\left(u_{\sigma(1)}\right) T^{\sigma(2)}\left(u_{\sigma(2)}\right) \cdots \tilde{f}_{\sigma(2 l-1) \sigma(2 l)} T^{\sigma(2 l-1)}\left(u_{\sigma(2 l-1)}\right) T^{\sigma(2 l)}\left(u_{\sigma(2 l)}\right) \\
& =\operatorname{Pf}\left[\tilde{f}_{i j} T^{(i)}\left(u_{i}\right) T^{(j)}\left(u_{j}\right)\right]_{i, j=1, \ldots, 2 l}=\operatorname{Pf}\left[\tilde{T}^{(i j)}\left(u_{i}, u_{j}\right)\right]_{i, j=1, \ldots, 2 l} .
\end{aligned}
$$

b) Observe that

$$
\begin{aligned}
\operatorname{Pf} & {\left[\tilde{T}^{(i j)}\left(u_{i}, u_{j}\right)\right]_{i, j=1, \ldots, 2 l}=\operatorname{Pf}\left[\sum_{\alpha_{i}, \alpha_{j}} \tilde{T}_{\alpha_{i}, \alpha_{j}}^{(i, j)} u_{i}^{\alpha_{i}} u_{j}^{\alpha_{j}}\right]_{i, j=1, \ldots, 2 l} } \\
& =\sum_{\sigma \in S_{2 l}^{\prime}} \operatorname{sgn}(\sigma) \sum_{\alpha_{i}, \alpha_{j}} \tilde{T}_{\alpha_{\sigma(1)}\left(\sigma(1), \alpha_{\sigma(2)}(2)\right)}^{\alpha_{\sigma(1)}} u_{\sigma(2)}^{\alpha_{\sigma(1)}} \cdots \tilde{T}_{\alpha_{\sigma(2 l-1)}, \alpha_{\sigma(2 l)}(\sigma(2 l-1), \sigma(2 l))}^{\alpha_{\sigma(2)}} u_{\sigma(2 l-1)}^{\alpha_{\sigma(2 l-1)}} u_{\sigma(2 l)}^{\alpha_{\sigma(2 l)}} \\
& =\sum_{\sigma \in S_{2 l}^{\prime}} \operatorname{sgn}(\sigma) \sum_{\alpha_{i}, \alpha_{j}} \tilde{T}_{\alpha_{\sigma(1)}\left(\sigma(1), \alpha_{\sigma(2)}\right.}^{(\sigma(2))} \cdots \tilde{T}_{\alpha_{\sigma(2 l-1)}(\sigma(2 l-1), \sigma(2 l))}^{(\sigma(2 l)} u_{1}^{\alpha_{1}} \ldots u_{2 l}^{\alpha_{2 l} l}=\sum_{\alpha} \operatorname{Pf}\left[\tilde{T}_{\alpha_{i}, \alpha_{j}}^{(i, j)}\right] u_{1}^{\alpha_{1}} \cdots u_{2 l}^{\alpha_{2 l}} .
\end{aligned}
$$

Therefore, the coefficient of $u_{1}^{\alpha_{1}} \cdots u_{2 l}^{\alpha_{2 l}}$ in (4.8) equals $\operatorname{Pf}\left[\tilde{T}_{\alpha_{i}, \alpha_{j}}^{(i, j)}\right]_{i, j=1, \ldots 2 l}$.

c) Let $A_{i}(u)=\sum_{N_{i} \geq j \geq M_{i}} A_{i, j} u^{j} \in \mathbb{C}\left[u, u^{-1}\right], i=1, \ldots, l, N_{i}, M_{i} \in \mathbb{Z}$. Then from (4.7),

$$
\begin{aligned}
T\left(u_{1}, \ldots, u_{l}\right) & =A_{1}\left(u_{1}\right) \varphi\left(u_{1}\right) \ldots A_{l}\left(u_{l}\right) \varphi\left(u_{l}\right)(1) \\
& =\sum_{N_{1} \geq j_{1} \geq M_{1}} \sum_{k_{1} \in \mathbb{Z}} A_{1, j_{1}} \varphi_{k_{1}} u_{1}^{j_{1}-k_{1}} \ldots \sum_{N_{l} \geq j_{l} \geq M_{l}} \sum_{k_{l} \in \mathbb{Z}} A_{l, j_{l}} \varphi_{k_{l}} u_{l}^{j_{l}-k_{l}}(1) \\
& =\sum_{\alpha \in \mathbb{Z}, l} \sum_{N_{1}-\alpha_{1} \geq k_{1} \geq M_{1}-\alpha_{1}} A_{1, \alpha_{1}+k_{1}} \varphi_{k_{1}} u_{1}^{\alpha_{1}} \ldots \sum_{N_{l}-\alpha_{l} \geq k_{l} \geq M_{l}-\alpha_{l}} A_{l, \alpha_{l}+k_{l}} \varphi_{k_{l}} u_{l}^{\alpha_{l}}(1) .
\end{aligned}
$$

Then the coefficient $T_{\alpha}$ of $u_{1}^{\alpha_{1}} \ldots u_{l}^{\alpha_{l}}$ is equal to $X_{1} \ldots X_{l}(1)$, where

$$
X_{j}=\sum_{N_{j}-\alpha_{j} \geq k \geq M_{j}-\alpha_{j}} A_{j, \alpha_{j}+k} \varphi_{k} .
$$

By Corollary 4.1 and Remark 4.1, the coefficient $T_{\alpha}$ is a tau-function of the BKP hierarchy. Since it is a finite linear combination of elements of the form $\varphi_{k_{1}} \ldots \varphi_{k_{l}}(1)$, it is a polynomial tau-function.

d) Following [11] Proposition 3, any polynomial tau-function has the form

$$
\tau=X_{1} \ldots X_{l}(1),
$$

where $\lambda=\left(\lambda_{1}>\lambda_{2}>\cdots>\lambda_{l} \geq 0\right)$ is a strict partition, and

$$
X_{j}=\sum_{N_{j} \geq i \geq-\lambda_{j}} b_{i, j} \varphi_{i}
$$

with $b_{i, j} \in \mathbb{C}$, and $b_{-\lambda_{j}, j} \neq 0, N_{j} \in \mathbb{Z}$ for $j=1, \ldots, l$. Fix $\left(\alpha_{1}, \ldots, \alpha_{l}\right) \in \mathbb{Z}^{l}$. In the definition of $T\left(u_{1}, \ldots, u_{l}\right)$ set the Laurent polynomial

$$
A_{j}(u)=\sum_{N_{j}+\alpha_{j} \geq k \geq \alpha_{j}-\lambda_{j}} b_{k-\alpha_{j}, j} u^{k}, \quad j=1, \ldots, l .
$$


POLYNOMIAL TAU-FUNCTIONS OF THE KP, BKP, AND THE $s$-COMPONENT KP HIERARCHIES

With such a choice of $A_{1}(u), \ldots, A_{l}(u)$ the operator (4.11) becomes exactly of the form (4.12), and the coefficient $T_{\alpha}$ coincides with the given polynomial tau-function $\tau$. In particular, take $\alpha_{1}=\cdots=$ $\alpha_{l}=0$ to represent $\tau$ as the constant coefficient of some $T\left(u_{1}, \ldots, u_{l}\right)$.

4.4. Remarks and corollaries. Theorem 4.1 implies several corollaries.

a) We proved that polynomial tau-functions are zero-modes of appropriate generating functions $T\left(u_{1}, \ldots, u_{l}\right)$, but changing back $A_{j}(u) \mapsto u^{\alpha_{j}} A_{j}(u)$ allows one to get any polynomial taufunction as a coefficient of a monomial $u_{1}^{\alpha_{1}} \ldots u_{l}^{\alpha_{l}}$ of any degree $\left(\alpha_{1}, \ldots, \alpha_{l}\right)$.

b) Consider non-zero Laurent polynomials $A_{1}(u), \ldots, A_{l}(u)$ in the definition of $T\left(u_{1}, \ldots, u_{l}\right)$. Due to remark above we can assume without loss of generality they are polynomials

$$
A_{j}(u)=b_{j} \sum_{i=0}^{N_{j}} a_{j, i} u^{i} \in \mathbb{C}[u]
$$

where $N_{j} \in \mathbb{Z}, b_{j}, a_{j, i} \in \mathbb{C}$, and $a_{j, 0}=1$. Note that there exists a collection of constants $\left\{c_{j, s}\right\} \subset \mathbb{C}$ such that

$$
\sum_{i=0}^{N_{j}} a_{j, i} u^{i}=\exp \left(\sum_{s=1}^{\infty} c_{j, s} u^{s}\right),
$$

in other words, $a_{j, i}=S_{i}\left(c_{j, 1}, c_{j, 2}, \ldots\right)$. Letting $\left(\tilde{t}_{1}, \tilde{t}_{2}, \tilde{t}_{3} \ldots\right)=\left(p_{1} / 2,0,3 p_{3} / 2,0, \ldots\right)$, we obtain by $(2.9)$

$$
\begin{aligned}
T^{(j)}\left(u_{j}\right) & =A_{j}\left(u_{j}\right) Q\left(u_{j}\right)=b_{j} \exp \left(\sum_{k \in \mathbb{N}} c_{j, k} u_{j}^{k}\right) \exp \left(\sum_{k \in \mathbb{N}} \tilde{t}_{k} u_{j}^{k}\right) \\
= & b_{j} \sum_{k \in \mathbb{N}} S_{k}\left(\tilde{t}_{1}+c_{j, 1}, \tilde{t}_{2}+c_{j, 2}, \ldots\right) u_{j}^{k},
\end{aligned}
$$

Then for $i<j$

$$
\tilde{T}^{(i, j)}=b_{i} b_{j}\left(1+2 \sum_{k \in \mathbb{N}}\left(\frac{u_{j}}{-u_{i}}\right)^{k}\right) \sum_{a, b \in \mathbb{N}} S_{a}\left(\tilde{t}+c_{i}\right) S_{b}\left(\tilde{t}+c_{j}\right) u_{i}^{a} u_{j}^{b} .
$$

and

$$
\tilde{T}_{a, b}^{(i, j)}=2 b_{i} b_{j} \chi_{a, b}\left(\tilde{t}+c_{i}, \tilde{t}+c_{j}\right)
$$

where

$$
\chi_{a, b}\left(\tilde{t}+c_{i}, \tilde{t}+c_{j}\right)=\frac{1}{2} S_{a}\left(\tilde{t}+c_{i}\right) S_{b}\left(\tilde{t}+c_{j}\right)+\sum_{k \in \mathbb{N}}(-1)^{k} S_{a-k}\left(\tilde{t}+c_{i}\right) S_{b+k}\left(\tilde{t}+c_{j}\right)
$$

for $a<b, \chi_{a, b}=-\chi_{b, a}$ for $a>b$, and $\chi_{a, a}=0$. By Theorem 4.1, polynomial tau-functions of the BKP hierarchy have the form

$$
T_{\alpha}=2^{2 l} \cdot\left(\prod_{k} b_{k}^{2}\right) \cdot \operatorname{Pf}\left[\chi_{a, b}\left(\tilde{t}+c_{i}, \tilde{t}+c_{j}\right)\right]_{i, j=1, \ldots, 2 l}
$$

for any choice of constant vectors $c_{j}=\left(c_{j, k}\right)_{k \in \mathbb{N}}$. This recovers the description of all polynomial tau-functions of the BKP hierarchy of [11] and, in particular, the result of You [18] that Schur Q-polynomials are polynomial tau-functions of the BKP hierarchy. 


\section{Polynomial $\tau$-Functions of the Bilinear $s$-COMPONENT KP Hierarchy}

In this section we consider the $s$-component KP hierarchy which generalizes Section 3 that treats the case $s=1$. Fix a positive integer $s$. Following [4], [9] and [12], consider $s$ copies $\Lambda^{(1)}, \ldots, \Lambda^{(s)}$ of the algebra of symmetric functions $\Lambda$. We assume that for $a \neq b$ the operators $H^{(a)}, E^{(a)}, H^{\perp(a)}$, $E^{\perp(a)}$, etc. acting on the copy $\Lambda^{(a)}$, commute with the operators $H^{(b)}, E^{(b)}, H^{\perp(b)}, E^{\perp(b)}$, etc., acting on $\Lambda^{(b)}$.

5.1. Fermionic fields on the $s$-component boson Fock space. Introduce the boson Fock space $\mathcal{B}^{\otimes s}$ as the tensor product of $s$ copies of the algebra $\mathcal{B}$ from Section 3.1:

$$
\mathcal{B}^{\otimes s}=\mathbb{C}\left[z_{a}, z_{a}^{-1} ; p_{1}^{(a)}, p_{2}^{(a)}, p_{3}^{(a)}, \ldots ; 1 \leq a \leq s\right]
$$

where $\left\{p_{k}^{(a)}\right\}_{a=1, \ldots, s}$ are collections of power sums in $s$ different sets of variables, $p_{k}^{(a)} \in \Lambda^{(a)}$. We have the charge decomposition

$$
\mathcal{B}^{\otimes s}=\oplus_{m \in \mathbb{Z}} \mathcal{B}^{\otimes s(m)},
$$

where

$$
\mathcal{B}^{\otimes s(m)}=\sum_{\substack{\left(m_{1}, \ldots, m_{s}\right) \in \mathbb{Z}^{s}, m_{1}+\cdots+m_{s}=m}} z_{1}^{m_{1}} \ldots z_{s}^{m_{s}} \mathbb{C}\left[p_{1}^{(a)}, p_{2}^{(a)}, p_{3}^{(a)}, \ldots\right] .
$$

Define the action of operators $R^{ \pm(a)}(u)$ on $\mathcal{B}^{\otimes s}$ by

$$
R^{ \pm(a)}(u)\left(z_{1}^{m_{1}} \ldots z_{s}^{m_{s}} f\right)=(-1)^{m_{1}+\cdots+m_{a-1}} z_{1}^{m_{1}} \ldots z_{a}^{m_{a} \pm 1} \ldots z_{s}^{m_{s}} u^{ \pm m_{a}} f
$$

for $\left(m_{1}, \ldots, m_{s}\right) \in \mathbb{Z}^{s}, f \in \mathbb{C}\left[p_{1}^{(a)}, p_{2}^{(a)}, p_{3}^{(a)}, \ldots ; 1 \leq a \leq s\right]$, which change the charge by \pm 1 .

It is straightforward to check the following lemma.

Lemma 5.1. For $a \neq b$,

$$
\begin{aligned}
R^{ \pm(a)}(u) R^{ \pm(b)}(v)+R^{ \pm(b)}(v) R^{ \pm(a)}(u) & =0, \\
R^{+(a)}(u) R^{-(b)}(v)+R^{-(b)}(v) R^{+(a)}(u) & =0, \\
v R^{ \pm(a)}(u) R^{ \pm(a)}(v)-u R^{ \pm(a)}(v) R^{ \pm(a)}(u) & =0, \\
u R^{+(a)}(u) R^{-(a)}(v)-v R^{-(a)}(v) R^{+(a)}(u) & =0 .
\end{aligned}
$$

Let $\mathcal{D}^{\otimes s}$ be the algebra of differential operators with polynomial coefficients acting on $\mathcal{B}^{\otimes s}$ (cf. Section 2.3). Similarly to (3.1), (3.2), define for $a \in\{1, \ldots, s\}$ a collection of $\mathcal{D}^{\otimes s}$-valued formal distributions

$$
\begin{aligned}
& \psi^{+(a)}(u)=R^{+(a)}(u) H^{(a)}(u) E^{(a) \perp}(-u), \\
& \psi^{-(a)}(u)=R^{-(a)}(u) E^{(a)}(-u) H^{(a) \perp}(u) .
\end{aligned}
$$

Define the operators $\left\{\psi_{i}^{ \pm(a)}\right\}_{i \in \mathbb{Z}+1 / 2}$ as the coefficients of expansions

$$
\psi^{ \pm(a)}(u)=\sum_{i \in \mathbb{Z}+1 / 2} \psi_{i}^{ \pm(a)} u^{-i-1 / 2}, \quad a=1, \ldots s .
$$

Using Lemmas 5.1 and 2.3, along the same lines as the proof of Proposition 3.1, we obtain the commutation relations for $\psi^{ \pm(a)}(u)$ :

Proposition 5.1. $\quad$ a) Formulae (5.1), (5.2) define quantum fields $\psi^{ \pm(a)}(u)$ of operators acting on $\mathcal{B}^{\otimes s}$. 
b) For all $a, b=1, \ldots, s$,

$$
\begin{aligned}
& \psi^{ \pm(a)}(u) \psi^{ \pm(b)}(v)+\psi^{ \pm(b)}(v) \psi^{ \pm(a)}(u)=0, \\
& \psi^{+(a)}(u) \psi^{-(b)}(v)+\psi^{-(b)}(v) \psi^{+(a)}(u)=\delta_{a, b} \delta(u, v) .
\end{aligned}
$$

Relations (5.3), (5.4) are equivalent to

$$
\begin{aligned}
& \psi_{k}^{ \pm(a)} \psi_{l}^{ \pm(b)}+\psi_{l}^{ \pm(b)} \psi_{k}^{ \pm(a)}=0, \\
& \psi_{k}^{+(a)} \psi_{l}^{-(b)}+\psi_{l}^{-(b)} \psi_{k}^{+(a)}=\delta_{k,-l} \delta_{a, b}, \quad k, l \in \mathbb{Z}+1 / 2 .
\end{aligned}
$$

c) Let $\varepsilon_{a b}=1$ if $b \leq a$ and $=-1$ if $b>a$, then

$$
z_{a} \psi_{n}^{ \pm(b)}=\varepsilon_{a b} \psi_{n \mp \delta_{a, b}}^{ \pm(b)} z_{a} .
$$

Remark 5.1. From (2.5), (2.6) the bosonic form of $\psi^{ \pm(a)}(u)$ is

$$
\begin{aligned}
& \psi^{+(a)}(u)=R^{+(a)}(u) \exp \left(\sum_{n \geq 1} \frac{p_{n}^{(a)}}{n} u^{n}\right) \exp \left(-\sum_{n \geq 1} \frac{\partial}{\partial p_{n}^{(a)}} \frac{1}{u^{n}}\right), \\
& \psi^{-(a)}(u)=R^{-(a)}(v) \exp \left(-\sum_{n \geq 1} \frac{p_{n}^{(a)}}{n} u^{n}\right) \exp \left(\sum_{n \geq 1} \frac{\partial}{\partial p_{n}^{(a)}} \frac{1}{u^{n}}\right)
\end{aligned}
$$

for $a=1, \ldots, s$. It follows that $\psi_{k-\ell_{a}}^{+(a)}\left(z_{1}^{\ell_{1}} \ldots z_{m}^{\ell_{m}}\right)=0$ and $\psi_{k+\ell_{a}}^{-(a)}\left(z_{1}^{\ell_{1}} \ldots z_{m}^{\ell_{m}}\right)=0$ for $k>0$.

5.2. Bilinear $s$-component KP identity. The bilinear s-component KP identity [9], [12] is the equation

$$
\Omega(\tau \otimes \tau)=0
$$

on a function $\tau=\tau\left(z_{1}, \ldots, z_{s}, p_{1}^{(1)}, \ldots, p_{1}^{(s)}, p_{2}^{(1)}, \ldots, p_{2}^{(s)}, \ldots\right)$ from the completion of $\mathcal{B}^{\otimes s(m)}$, where

$$
\Omega=\sum_{a=1}^{s} \Omega^{(a)}, \quad \Omega^{(a)}=\sum_{k \in \mathbb{Z}+\frac{1}{2}} \psi_{k}^{+(a)} \otimes \psi_{-k}^{-(a)} .
$$

Non-zero solutions of (5.8) are called tau-functions of charge $m$ of the $s$-component KP hierarchy. We say that a non-zero solution $\tau$ of (5.8) is a polynomial tau-function if $\tau=\sum_{a \in \mathbb{Z}^{s},|a|=m} z^{a} f_{a} \in \mathcal{B}^{\otimes s}(m)$, where $f_{a}$ are polynomial functions in the variables $\left(p_{1}^{(1)}, \ldots, p_{1}^{(s)}, p_{2}^{(1)}, \ldots, p_{2}^{(s)}, \ldots\right)$ and the sum is finite. The vacuum vector 1 , as well as $z^{a}$, are obviously tau-functions of the $s$-component $\mathrm{KP}$ hierarchy. Similarly to Section 3.2, we use a family of commuting with $\Omega$ operators to construct other tau-functions.

Remark 5.2. Note that, by (5.7), $z_{i} \otimes z_{i}$ commutes with $\Omega$ for every $1 \leq i \leq s$. Thus, if

$$
\tau=\sum_{m \in \mathbb{Z}^{s},|m|=l} z^{m} \tau_{m}
$$

is a tau-function of the $s$-component $\mathrm{KP}$ hierarchy of charge $l$, then for $a=\left(a_{1}, \ldots, a_{s}\right) \in \mathbb{Z}^{s}$,

$$
\sum_{m \in \mathbb{Z}^{s},|m|=l}(-1)^{\sum_{i=2}^{s}(-1)^{a_{i}\left(m_{1}+\cdots+m_{i-1}\right)}} z^{m+a} \tau_{m}
$$

is its tau-function of charge $l+|a|$. 
Lemma 5.2. Let $X^{(a)}=\sum_{k>M} A_{k}^{(a)} \psi_{k}^{+(a)}$, where $A_{k}^{(a)} \in \mathbb{C}, M \in \mathbb{Z}, a=1, \ldots, s$. Let $X=$ $\sum_{a=1}^{s} X^{(a)}$. Then

$$
\begin{aligned}
& \left(X^{(a)}\right)^{2}=0, \quad X^{2}=0, \\
& X^{(a)} X^{(b)}+X^{(b)} X^{(a)}=0 \quad \text { for } \quad a \neq b, \\
& \psi_{k}^{-(a)} X^{(b)}+X^{(b)} \psi_{k}^{-(a)}=\delta_{a, b} A_{k}^{(b)} .
\end{aligned}
$$

Proof. Note that $X^{(a)},\left(X^{(a)}\right)^{2}, X$ and $X^{2}$ are well-defined operators by Proposition 5.1 (a). Identity $\left(X^{(a)}\right)^{2}=0$ follows from the KP case, Lemma 3.1. Commutation relations for $a \neq b$ follow from (5.5), (5.6). Finally,

$$
X^{2}=\sum_{a=1}^{s}\left(X^{(a)}\right)^{2}+\sum_{s \geq a>b \geq 1}\left(X^{(a)} X^{(b)}+X^{(b)} X^{(a)}\right)=0 .
$$

Lemma 5.3. Let $X^{(a)}=\sum_{k>M} A_{k}^{(a)} \psi_{k}^{+(a)}$ where $A_{k}^{(a)} \in \mathbb{C}, M \in \mathbb{Z}, a=1, \ldots, s$. Let $X=$ $\sum_{a=1}^{s} X^{(a)}$. Then

$$
\Omega\left(X^{(a)} \otimes X^{(a)}\right)=\left(X^{(a)} \otimes X^{(a)}\right) \Omega \quad \text { and } \quad \Omega(X \otimes X)=(X \otimes X) \Omega .
$$

Proof. For obvious reasons $\Omega^{(b)}\left(X^{(a)} \otimes X^{(a)}\right)=\left(X^{(a)} \otimes X^{(a)}\right) \Omega^{(b)}$ for $a \neq b$, while $\Omega^{(a)}\left(X^{(a)} \otimes X^{(a)}\right)=$ $\left(X^{(a)} \otimes X^{(a)}\right) \Omega^{(a)}$ from the KP case Lemma 3.2. Then the statement follows.

Corollary 5.1. Let $\tau \in \mathcal{B}^{\otimes s}$ be a tau-function of charge $m$ of the s-component KP hierarchy. Let $X^{(a)}=\sum_{k>M} A_{k}^{(a)} \psi_{k}^{+(a)}$ where $A_{k}^{(a)} \in \mathbb{C}, M \in \mathbb{Z}, a=1, \ldots, s$. Let $X=\sum_{a=1}^{s} X^{(a)}$. Then $\tau^{\prime(a)}=X^{(a)} \tau$ and $\tau^{\prime}=X \tau$ are tau-functions of this hierarchy of charge $m+1$.

5.3. Generating function for polynomial tau-functions of the $s$-component KP hierarchy. For $1 \leq a_{i} \leq s, i=1, \ldots, l$ let

$$
G^{\left(a_{l}, \ldots, a_{1}\right)}\left(u_{1}, \ldots, u_{l}\right)=\prod_{i<j}\left(u_{j}-u_{i}\right)^{\delta_{a_{i}, a_{j}}} \prod_{i=1}^{l} H^{\left(a_{i}\right)}\left(u_{i}\right)
$$

be a power series in the variables $u_{1}, \ldots, u_{l}$ with coefficients in $\mathcal{B}^{\otimes s}$.

Definition 5.1. Define the function $\varepsilon\left(a_{l}, \ldots, a_{1}\right)$ on the sets of finite sequences of natural numbers with values in \pm 1 by the following recurrent formula: Set

$$
\varepsilon(a)=1, \quad \text { and } \quad \varepsilon\left(a, a_{l}, \ldots, a_{1}\right)=(-1)^{s\left(a, a_{l}, \ldots, a_{1}\right)} \varepsilon\left(a_{l}, \ldots, a_{1}\right),
$$

where $s\left(a, a_{l}, \ldots, a_{1}\right)=\#\left\{a_{i} \mid a_{i}<a, i=1, \ldots, l\right\}$.

The following lemma is straightforward.

Lemma 5.4.

a)

$$
\varepsilon(\overbrace{s, \ldots, s}^{m_{s}}, \ldots, \overbrace{1, \ldots, 1}^{m_{1}})=(-1)^{\sum_{i<j} m_{i} m_{j}}
$$

b)

$$
s\left(a, a_{l}, \ldots, a_{1}\right)=\sum_{r=1}^{a-1} m_{r},
$$

where $m_{r}$ is the multiplicity of $r$ in the sequence $\left(a_{l}, \ldots, a_{1}\right)$. 
c) Let $\rho \in S_{l}$ be a permutation which does not change the order of the $a_{i}$ which are the same. Then $\varepsilon\left(a_{\rho(l)}, \ldots a_{\rho(1)}\right)=\operatorname{sgn}(\rho) \varepsilon\left(a_{l}, \ldots, a_{1}\right)$.

Proposition 5.2. Let $1 \leq a_{i} \leq s, i=1, \ldots, l$. Then

$$
\psi^{+\left(a_{l}\right)}\left(u_{l}\right) \ldots \psi^{+\left(a_{1}\right)}\left(u_{1}\right)(1)=\varepsilon\left(a_{l}, \ldots, a_{1}\right) z_{a_{1}} \ldots z_{a_{l}} G^{\left(a_{l}, \ldots, a_{1}\right)}\left(u_{1}, \ldots, u_{l}\right) .
$$

Proof. By induction on $l$. Observe that $\psi^{+(a)}(u)(1)=H^{(a)}(u)$. Assume that for $\left(a_{1}, \ldots, a_{l}\right)$ formula (5.9) holds. Let $\left(a_{l}, \ldots, a_{1}\right)$ be a permutation of

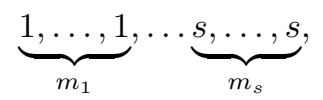

where $m_{i} \geq 0$ and $m_{1}+\cdots+m_{s}=l$. Then from (5.1), Lemma $5.4 \mathrm{~b}$ ) and Lemma 2.3 we obtain

$$
\begin{aligned}
& \psi^{+(a)}\left(u_{l+1}\right) \psi^{+\left(a_{l}\right)}\left(u_{l}\right) \ldots \psi^{+\left(a_{1}\right)}\left(u_{1}\right)(1)= \\
& =\psi^{+(a)}\left(u_{l+1}\right)\left(\varepsilon\left(a_{l}, \ldots, a_{1}\right) z_{1}^{m_{1}} \ldots z_{l}^{m_{l}} \prod_{i<j}\left(u_{j}-u_{i}\right)^{\delta_{a_{i}, a_{j}}} \prod_{i=1}^{l} H^{\left(a_{i}\right)}\left(u_{i}\right)\right) \\
& =\varepsilon\left(a_{l}, \ldots, a_{1}\right)(-1)^{\sum_{r=1}^{a-1} m_{r}} z_{1}^{m_{1}} \ldots z_{a}^{m_{a}+1} \ldots z_{l}^{m_{l}} u_{l+1}^{m_{a}} \prod_{i=1}^{l}\left(1-\frac{u_{i}}{u_{l+1}}\right)^{\delta_{a_{i}, a}} \prod_{i<j}\left(u_{j}-u_{i}\right)^{\delta_{a_{i}, a_{j}}} \prod_{i=1}^{l+1} H^{\left(a_{i}\right)}\left(u_{i}\right) \\
& =\varepsilon\left(a, a_{l}, \ldots, a_{1}\right) z_{a_{1}} \ldots z_{a_{l+1}} G^{\left(a, a_{1}, \ldots, a_{l}\right)}\left(u_{1}, \ldots, u_{l+1}\right),
\end{aligned}
$$

which proves the statement by the inductive assumption.

From Proposition 5.2 it follows that in the expansion

$$
G^{\left(a_{l}, \ldots, a_{1}\right)}\left(u_{1}, \ldots, u_{l}\right)=\sum_{\alpha=\left(\alpha_{1}, \ldots, \alpha_{l}\right) \in \mathbb{Z}_{+}^{l}} G_{\alpha}^{\left(a_{l}, \ldots, a_{1}\right)} u_{1}^{\alpha_{1}} \ldots u_{l}^{\alpha_{l}}
$$

one has

$$
G_{\alpha}^{\left(a_{l}, \ldots, a_{1}\right)}=\varepsilon\left(a_{l}, \ldots, a_{1}\right) z_{a_{1}}^{-1} \ldots z_{a_{l}}^{-1} \psi_{-\alpha_{l}-1 / 2}^{+\left(a_{l}\right)} \ldots \psi_{-\alpha_{1}-1 / 2}^{+\left(a_{1}\right)}(1) .
$$

As in Section 3.3, we introduce a collection of non-zero formal Laurent series $A_{i}^{(r)}(u) \in \mathbb{C}((u)), r=$ $1, \ldots, s, i=1, \ldots, l$. Define the $\mathcal{B}^{\otimes s}$-valued Laurent series $T_{i}^{(r)}(u)=A_{i}^{(r)}(u) H^{(r)}(u) \in \mathcal{B}^{\otimes s(0)}((u))$. Set

$$
\begin{aligned}
T^{\left(a_{l}, \ldots, a_{1}\right)}\left(u_{1}, \ldots, u_{l}\right) & =\prod_{j=1}^{l} A_{j}^{\left(a_{j}\right)}\left(u_{j}\right) G^{\left(a_{l}, \ldots, a_{1}\right)}\left(u_{1}, \ldots, u_{l}\right) \\
& =\prod_{i<j}\left(u_{j}-u_{i}\right)^{\delta_{a_{i}, a_{j}}} \prod_{j=1}^{l} T_{j}^{\left(a_{j}\right)}\left(u_{j}\right) \in \mathcal{B}^{\otimes s(0)}\left[\left[u_{1}, \ldots, u_{l}\right]\right]\left[u_{1}^{-1}, \ldots, u_{l}^{-1}\right] .
\end{aligned}
$$

Set also

$$
T\left(u_{1}, \ldots, u_{l}\right)=\sum_{a_{1}, \ldots, a_{l}=1}^{s} \varepsilon\left(a_{l}, \ldots, a_{1}\right) z_{a_{1}} \ldots z_{a_{l}} T^{\left(a_{l}, \ldots, a_{1}\right)}\left(u_{1}, \ldots, u_{l}\right),
$$


Let $T_{i ; k}^{(r)}$ and $T_{\alpha}^{\left(a_{l}, \ldots, a_{1}\right)}, T_{\alpha}$ be the coefficients of the expansions

$$
\begin{aligned}
T_{i}^{(r)}(u) & =\sum_{k \in \mathbb{Z}} T_{i ; k}^{(r)} u^{k}, \\
T^{\left(a_{l}, \ldots, a_{1}\right)}\left(u_{1}, \ldots, u_{l}\right) & =\sum_{\alpha \in \mathbb{Z}^{l}} T_{\alpha}^{\left(a_{l}, \ldots, a_{1}\right)} u_{1}^{\alpha_{1}} \ldots u_{l}^{\alpha_{l}}, \\
T\left(u_{1}, \ldots, u_{l}\right) & =\sum_{\alpha \in \mathbb{Z}^{l}} T_{\alpha} u_{1}^{\alpha_{1}} \ldots u_{l}^{\alpha_{l}} .
\end{aligned}
$$

In Section 3.3 we expressed the generating function of the KP tau-functions as a determinant. We will show that the generating function in the multicomponent $\mathrm{KP}$ case $T^{\left(a_{l}, \ldots, a_{1}\right)}\left(u_{1}, \ldots, u_{l}\right)$ is a product of appropriate determinants of the form (3.13), First, we illustrate this result, inspired by [12] Theorem 4, with an example, and then formulate and prove the general statement. We also describe polynomial tau-functions of the bilinear $s$-component KP identity as coefficients of $T\left(u_{1}, \ldots, u_{l}\right)$.

Example 5.1. We have

$$
\begin{aligned}
T^{(b, b, a, b, a)}\left(u_{1}, u_{2}, u_{3}, u_{4}, u_{5}\right)= & \left(u_{3}-u_{1}\right) T_{1}^{(a)}\left(u_{1}\right) T_{3}^{(a)}\left(u_{3}\right) \\
& \times\left(u_{5}-u_{4}\right)\left(u_{5}-u_{2}\right)\left(u_{4}-u_{2}\right) T_{2}^{(b)}\left(u_{2}\right) T_{4}^{(b)}\left(u_{4}\right) T_{5}^{(b)}\left(u_{5}\right) \\
= & \operatorname{det}\left[u_{i}^{j-1} T_{i}^{(b)}\left(u_{i}\right)\right]_{\substack{i=2,4,5 \\
j=1,2,3}} \operatorname{det}\left[u_{i}^{j-1} T_{i}^{(a)}\left(u_{i}\right)\right]_{\substack{i=1,3 \\
j=1,2}} .
\end{aligned}
$$

More generally, for any choice of parameters $\left(a_{l}, \ldots, a_{1}\right), a_{i} \in\{1, \ldots, s\}$, define a partition of the set $\{1, \ldots, l\}=I_{1} \sqcup \cdots \sqcup I_{s}$ into the subsets $I_{1}, \ldots, I_{s}$, where $I_{r}=\left\{i \in\{1, \ldots, l\} \mid a_{i}=r\right\}$. If $\left|I_{r}\right|=m_{r}$, then $m_{1}+\cdots+m_{r}=l$. For example, for the choice $\left(a_{4}, a_{3}, a_{2}, a_{1}\right)=(1,3,3,5)$, one gets $I_{1}=\{4\}, I_{3}=\{2,3\}, I_{5}=\{1\}$, and the rest of $I_{r}$ 's are empty.

Theorem 5.1. $\quad$ a) For any choice of $a_{i} \in\{1, \ldots, s\}, i=1, \ldots, l$, we have

$$
T^{\left(a_{l}, \ldots, a_{1}\right)}\left(u_{1}, \ldots, u_{l}\right)=\prod_{r=1}^{s} \operatorname{det}\left[u_{i}^{j-1} T_{i}^{(r)}\left(u_{i}\right)\right]_{i \in I_{r}, j=1, \ldots, m_{r}} .
$$

b) For any $\left(\alpha_{1}, \ldots, \alpha_{l}\right) \in \mathbb{Z}^{l}$ the coefficient of the monomial $u_{1}^{\alpha_{1}} \ldots u_{l}^{\alpha_{l}}$ in (5.10) is given by

$$
T_{\alpha}^{\left(a_{l}, \ldots, a_{1}\right)}=\prod_{r=1}^{s} \operatorname{det}\left[T_{i ; \alpha_{i}+1-j}^{(r)}\right]_{i \in I_{r}, j=1, \ldots, m_{r}} .
$$

c) For any $\left(\alpha_{1}, \ldots, \alpha_{l}\right) \in \mathbb{Z}^{l}$ the coefficient $T_{\alpha}^{\left(a_{l}, \ldots, a_{1}\right)}$ of the monomial $u_{1}^{\alpha_{1}} \ldots u_{l}^{\alpha_{l}}$ in (5.12) is a tau-function of the s-component KP hierarchy. If $A_{i}^{(r)}(u) \in \mathbb{C}\left[u, u^{-1}\right]$ are Laurent polynomials for all $i=1, \ldots, l, r=1, \ldots, s$, then the element $T_{\alpha}^{\left(a_{l}, \ldots, a_{1}\right)}$ is a polynomial tau-function.

d) For any $\left(\alpha_{1}, \ldots, \alpha_{l}\right) \in \mathbb{Z}^{l}$ the coefficient $T_{\alpha}$ of the monomial $u_{1}^{\alpha_{1}} \ldots u_{l}^{\alpha_{l}}$ (5.13) is a taufunction of the s-component KP hierarchy. If $A_{i}^{(r)}(u) \in \mathbb{C}\left[u, u^{-1}\right]$ are Laurent polynomials for all $i=1, \ldots, l, r=1, \ldots, s$, then the element $T_{\alpha}$ is a polynomial tau-function.

e) Let $\tau$ be a polynomial $\tau$-function of the s-component KP hierarchy. Then there exists a collection of complex-valued Laurent polynomials $A_{i}^{(r)}(u) \in \mathbb{C}\left[u, u^{-1}\right] i=1, \ldots, l, r=1, \ldots, s$, such that $\tau$ is a zero-mode of the Laurent series expansion of $T\left(u_{1}, \ldots, u_{l}\right)$ in (5.11). 
Proof. a) Observe that

$$
\prod_{i<j}\left(u_{j}-u_{i}\right)^{\delta_{a_{i}, a_{j}}}=\prod_{r=1}^{s} \prod_{\substack{i<j \\ i, j \in I_{r}}}\left(u_{j}-u_{i}\right)=\prod_{r=1}^{s} \operatorname{det}\left[u_{i}^{j-1}\right]_{i \in I_{r}, j=1, \ldots, m_{r}}
$$

and

$$
\prod_{j=1}^{l} T_{j}^{\left(a_{j}\right)}\left(u_{j}\right)=\prod_{r=1}^{s} \prod_{i \in I_{r}} T_{i}^{(r)}\left(u_{i}\right)
$$

Then

$T^{\left(a_{l}, \ldots, a_{1}\right)}\left(u_{1}, \ldots, u_{l}\right)=\prod_{r=1}^{s}\left(\operatorname{det}\left[u_{i}^{j-1}\right]_{i \in I_{r}, j=1, \ldots, m_{r}} \prod_{i \in I_{r}} T_{i}^{(r)}\left(u_{i}\right)\right)=\prod_{r=1}^{s} \operatorname{det}\left[u_{i}^{j-1} T_{i}^{(r)}\left(u_{i}\right)\right]_{\substack{i \in I_{r}, j=1, \ldots, m_{r}}}$.

b) This follows from a) and (3.14).

c) Let $A_{j}^{(r)}(u)=\sum_{k \geq M_{j}^{(r)}} A_{j, k-1 / 2}^{(r)} u^{k}$, where $A_{j, k-1 / 2}^{(r)} \in \mathbb{C}, M_{j}^{(r)} \in \mathbb{Z}, r=1, \ldots, s, j=1, \ldots, l$, $k \in \mathbb{Z}$. By Propostion 5.9 and definition (5.10),

$$
\begin{aligned}
A_{l}^{\left(a_{l}\right)}\left(u_{l}\right) \ldots A_{1}^{\left(a_{1}\right)}\left(u_{1}\right) \psi^{+\left(a_{l}\right)}\left(u_{l}\right) \ldots \psi^{+\left(a_{1}\right)}\left(u_{1}\right)(1) \\
\quad=\varepsilon\left(a_{l}, \ldots, a_{1}\right) z_{a_{1}} \ldots z_{a_{l}} T^{\left(a_{l}, \ldots, a_{1}\right)}\left(u_{1}, \ldots, u_{l}\right) .
\end{aligned}
$$

Then the coefficient of $u_{1}^{\alpha_{1}} \ldots u_{l}^{\alpha_{l}}$ in $T^{\left(a_{l}, \ldots, a_{1}\right)}\left(u_{1}, \ldots, u_{l}\right)$ can be represented in the form $T_{\alpha}^{\left(a_{l}, \ldots, a_{1}\right)}=$ $\varepsilon\left(a_{l}, \ldots, a_{1}\right) z_{a_{1}}^{-1} \ldots z_{a_{l}}^{-1} X_{l}^{\left(a_{l}\right)} \ldots X_{1}^{\left(a_{1}\right)}(1)$ with

$$
X_{j}^{(a)}=\sum_{i \geq M_{j}^{(a)}-\alpha_{j}-1 / 2} A_{j, \alpha_{j}+i}^{(a)} \psi_{i}^{+(a)} .
$$

By Corollary 5.1, it is a tau-function of the $s$-component KP hierarchy.

d) Similarly, the coefficient of $u_{1}^{\alpha_{1}} \ldots u_{l}^{\alpha_{l}}$ in $T\left(u_{1}, \ldots, u_{l}\right)$ is of the form

$$
\sum_{a_{1}, \ldots, a_{l}=1}^{s} \varepsilon\left(a_{1}, \ldots, a_{l}\right) z_{a_{1}} \ldots z_{a_{l}} T_{\alpha}^{\left(a_{l}, \ldots, a_{1}\right)}=\sum_{a_{1}, \ldots, a_{l}=1}^{s} X_{l}^{\left(a_{l}\right)} \ldots X_{1}^{\left(a_{1}\right)}(1)=X_{l} \ldots X_{1}(1),
$$

where $X_{j}=\sum_{a=1}^{s} X_{j}^{(a)}$. By Corollary 5.1, this coefficient is a tau-function of the $s$-component KP hierarchy.

If all of $A_{i}^{(r)}(u)$ are Laurent polynomials, the sum (5.15) has finitely many non-zero terms, and the coefficient of $u_{1}^{\alpha_{1}} \ldots u_{l}^{\alpha_{l}}$ is a finite linear combination of elements of the form $\psi_{k_{1}}^{+\left(a_{1}\right)} \ldots \psi_{k_{l}}^{+\left(a_{l}\right)}(1)$, hence it is a polynomial $\tau$-function.

e) Following [12], [9], by the boson-fermion correspondence we identify $\mathcal{B}^{\otimes s}$ with the semi-infinite wedge space $\Lambda^{\frac{1}{2}}\left(\mathbb{C}^{\infty}\right)$, where $\mathbb{C}^{\infty}=\oplus_{a=1}^{s} \oplus_{j \in \mathbb{Z}} \mathbb{C}_{j}^{(a)}$. As argued in [12], without loss of generality we can consider polynomial $\tau$-functions of the form

$$
\tau=f_{l} \wedge \cdots \wedge f_{1} \wedge|0\rangle,
$$

where

$$
f_{j}=\sum_{a=1}^{s} f_{j}^{(a)}, \quad f_{j}^{(a)}=\sum_{k=1}^{M_{j}^{(a)}} b_{k j}^{(a)} e_{k}^{(a)}, \quad j=1, \ldots, m, \quad a=1, \ldots, s,
$$

and

$$
|0\rangle=\left(e_{0}^{(1)} \wedge e_{-1}^{(1)} \wedge e_{-2}^{(1)} \wedge \ldots\right) \wedge \cdots \wedge\left(e_{0}^{(s)} \wedge e_{-1}^{(s)} \wedge e_{-2}^{(s)} \wedge \ldots\right),
$$


since under the boson-fermion correspondence the image of any other $\tau$-function in $\mathcal{B}^{\otimes s}$ is a $z^{a}$ multiple of the image of a function of the form (5.16): $\tau^{\prime}=z^{a} \tau$.

Using that $\psi_{-k+1 / 2}^{+(a)}\left(e_{i_{1}}^{\left(c_{1}\right)} \wedge e_{i_{2}}^{\left(c_{2}\right)} \ldots\right)=e_{k}^{(a)} \wedge e_{i_{1}}^{\left(c_{1}\right)} \wedge e_{i_{2}}^{\left(c_{2}\right)} \ldots$ we can write

$$
f_{l} \wedge \cdots \wedge f_{1} \wedge|0\rangle=\sum_{a_{1}, \ldots, a_{l}=1}^{s} Y_{l}^{\left(a_{l}\right)} \ldots Y_{1}^{\left(a_{1}\right)}|0\rangle=Y_{l} \ldots Y_{1}|0\rangle
$$

where

$$
Y_{j}^{(a)}=\sum_{-M_{j}^{(a)}+1 / 2 \leq k \leq-1 / 2} b_{-k+1 / 2, j}^{(a)} \psi_{k}^{+(a)}, \quad j=1, \ldots, l, \quad \text { and } \quad Y_{j}=\sum_{a=1}^{s} Y_{j}^{(a)} .
$$

Set

$$
A_{j}^{(a)}(u)=u^{\alpha_{j}} \sum_{-M_{j}^{(a)}+1 / 2 \leq k \leq-1 / 2} b_{-k+1 / 2, j}^{(a)} u^{k-1 / 2} \quad a=1, \ldots, s,
$$

Consider $T^{\left(a_{l}, \ldots, a_{1}\right)}\left(u_{1}, \ldots, u_{l}\right)$ defined with the choice (5.18) of Laurent series multipliers. Then $X_{j}^{(a)}$ in (5.15) coincides with $Y_{j}^{(a)}$ in (5.17).

Hence, the coefficient of $u_{1}^{\alpha_{1}} \ldots u_{l}^{\alpha_{l}}$ in $T^{\left(a_{1}, \ldots, a_{l}\right)}\left(u_{1}, \ldots, u_{l}\right)$ coincides with

$$
\varepsilon\left(a_{l}, \ldots, a_{1}\right) z_{a_{1}}^{-1} \ldots z_{a_{l}}^{-1} Y_{l}^{\left(a_{l}\right)} \ldots Y_{1}^{\left(a_{1}\right)}|0\rangle=\varepsilon\left(a_{l}, \ldots, a_{1}\right) z_{a_{1}}^{-1} \ldots z_{a_{l}}^{-1} f_{l}^{\left(a_{l}\right)} \wedge \cdots \wedge f_{1}^{\left(a_{1}\right)} \wedge|0\rangle,
$$

while the coefficient of $u_{1}^{\alpha_{1}} \ldots u_{l}^{\alpha_{l}}$ in (5.11) is $\sum_{a} f_{l}^{\left(a_{l}\right)} \wedge \cdots \wedge f_{1}^{\left(a_{1}\right)} \wedge|0\rangle=f_{l} \wedge \cdots \wedge f_{1} \wedge|0\rangle=\tau$. In particular, consider $\alpha_{1}=\cdots=\alpha_{l}=0$ to realize $\tau$ as a zero-mode of $T\left(u_{1}, \ldots, u_{l}\right)$.

Remark 5.3. Consider $s$ copies of the KP-hierarchy and let $\tau^{(1)}, \ldots, \tau^{(s)}$ be their tau-functions. Then the product $\tau^{(1)} \ldots \tau^{(s)}$ is a tau-function of the $s$-component KP hierarchy. This follows from the definition of the tau-function of the $s$-component KP hierarchy.

We now want to generalize Theorem $5.1 \mathrm{~b}$ ) and c). Let $\tau=\sum_{m \in \mathbb{Z}^{s},|m|=l} z^{m} \tau_{m} \in \mathcal{B}^{\otimes s}(l)$ be taufunction of charge $l$ of the $s$-component KP hierarchy. Then $\tau_{m}$ is the coefficient of the monomial $u_{1}^{\alpha_{1}} u_{2}^{\alpha_{2}} \cdots u_{l}^{\alpha_{l}} z_{1}^{m_{1}} z_{2}^{m_{2}} \cdots z_{s}^{m_{s}}$ in $T\left(u_{1}, u_{2}, \ldots, u_{l}\right)$. To obtain this coefficient, one has to sum all $\varepsilon\left(a_{l}, \ldots a_{1}\right) T^{\left(a_{l}, \ldots, a_{1}\right)}\left(u_{1}, \ldots u_{1}\right)$, where $\left(a_{1}, \ldots, a_{l}\right)$ is a permutation of $(\overbrace{1, \ldots, 1}^{m_{1} \text { times }}, \ldots, \overbrace{s, \ldots, s}^{m_{s} \text { times }})$. Using Theorem 5.1 a), we find that

$$
T^{(s, \ldots, s \ldots, 1 \ldots, 1)}\left(u_{1}, \ldots, u_{l}\right)=\prod_{r=1}^{s} \operatorname{det}\left[u_{i}^{j-1} T_{i}^{(r)}\left(u_{i}\right)\right]_{m_{1}+\cdots+m_{r-1}<i \leq m_{1}+\cdots+m_{r}, j=1, \ldots, m_{r}} .
$$

We want to write this as one determinant. Introduce, for $m \in \mathbb{Z}^{s}$, the function $\sigma_{m}(x)$ on the interval $[0, l]$, which is piece-wise linear of slope $45^{\circ}$, continuous at all points except for points $\left\{m_{1}, m_{1}+m_{2}, \ldots, m_{1}+\cdots+m_{s-1}\right\}$, and

$$
\sigma_{m}(0)=0, \quad \sigma_{m}\left(m_{1}+\cdots+m_{r}\right)=m_{r} \quad \text { for } \quad r=1, \ldots, s .
$$

Consider the subgroup $S_{l}^{\prime}=S_{m_{1}} \times S_{m_{2}} \times \cdots \times S_{m_{s}}$ of $S_{l}$. Using Lemma 5.4 a) and the notation

$$
\left(a_{l}, \ldots, a_{1}\right)=(\overbrace{s, \ldots, s}^{m_{s} \text { times }}, \ldots, \overbrace{1, \ldots, 1}^{m_{1} \text { times }})
$$


we obtain

$$
\varepsilon\left(a_{l}, \ldots a_{1}\right) T^{\left(a_{l}, \ldots, a_{1}\right)}\left(u_{1}, \ldots, u_{l}\right)=(-1)^{\sum_{i<j} m_{i} m_{j}} \sum_{\pi \in S_{l}^{\prime}} \operatorname{sgn}(\pi) \prod_{i=1}^{l} u_{\pi(i)}^{\sigma_{m}(i)-1} T_{\pi(i)}^{\left(a_{i}\right)}\left(u_{\pi(i)}\right) .
$$

Next, let $\rho \in S_{l}$ be a permutation which does not change the order of the $a_{i}$ which are the same. Then, by Lemma 5.4c), $\varepsilon\left(a_{\rho(l)}, \ldots a_{\rho(1)}\right)=\operatorname{sgn}(\rho) \varepsilon\left(a_{l}, \ldots, a_{1}\right)$, and $\left.T^{\left(a_{\rho(l)}, \ldots, a_{\rho(1)}\right)}\left(u_{1}, \ldots, u_{l}\right)\right)=$ $T^{\left(a_{l}, \ldots, a_{1}\right)}\left(u_{\rho(1)}, \ldots, u_{\rho(l)}\right)$.

Thus

$$
\begin{aligned}
& \varepsilon\left(a_{l}, \ldots, a_{1}\right) \varepsilon\left(a_{\rho(l)}, \ldots a_{\rho(1)}\right) T^{\left(a_{\rho(l)}, \ldots, a_{\rho(1)}\right)}\left(u_{1}, \ldots, u_{l}\right)=\operatorname{sgn}(\rho) T^{\left(a_{l}, \ldots, a_{1}\right)}\left(u_{\rho(1)}, \ldots, u_{\rho(l)}\right) \\
= & \operatorname{sgn}(\rho) \sum_{\rho \pi \mid \pi \in S_{l}^{\prime}} \operatorname{sgn}(\pi) \prod_{i=1}^{l} u_{\rho \pi(i)}^{\sigma_{m}(i)-1} T_{\rho \pi(i)}^{\left(a_{i}\right)}\left(u_{\rho \pi(i)}\right) .
\end{aligned}
$$

Hence to obtain the coefficient of $z_{1}^{m_{1}} \cdots z_{s}^{m_{s}}$ of $\varepsilon\left(a_{l}, \ldots, a_{1}\right) T\left(u_{1}, \ldots, u_{s}\right)$, we have to sum over all such permutations $\rho$ that do not change the order of the $a_{i}$ that are the same. Therefore this coefficient is equal to

$$
\sum_{\rho} \sum_{\rho \pi \mid \pi \in S_{l}^{\prime}} \operatorname{sgn}(\rho \pi) \prod_{i=1}^{l} u_{\rho \pi(i)}^{\sigma_{m}(i)-1} T_{\rho \pi(i)}^{\left(a_{i}\right)}\left(u_{\rho \pi(i)}\right) .
$$

This means that we sum over all permutations of $S_{l}$ and hence that the coefficient of $z_{1}^{m_{1}} \cdots z_{s}^{m_{s}}$ of $\varepsilon\left(a_{l}, \ldots, a_{1}\right) T\left(u_{1}, \ldots, u_{s}\right)$ is equal to

$$
\sum_{\pi \in S_{l}} \operatorname{sgn}(\pi) \prod_{i=1}^{l} u_{\pi(i)}^{\sigma_{m}(i)-1} T_{\pi(i)}^{\left(a_{i}\right)}\left(u_{\pi(i)}\right)=\operatorname{det}\left[u_{i}^{\sigma_{m}(j)-1} T_{i}^{\left(a_{j}\right)}\left(u_{i}\right)\right]_{i, j=1, \ldots, l} .
$$

Thus we have obtained the following

Theorem 5.2. For any $\left(\alpha_{1}, \ldots, \alpha_{l}\right) \in \mathbb{Z}^{l}$ the coefficient of the monomial $u_{1}^{\alpha_{1}} \ldots u_{l}^{\alpha_{l}}$ in (5.11) is a tau-function $\tau=\sum_{m \in \mathbb{Z}^{s},|m|=l} z^{m} \tau_{m}$ of charge $l$ of the s-component KP hierarchy. In particular, $\tau_{m}$ is the coefficient of $u_{1}^{\alpha_{1}} \ldots u_{l}^{\alpha_{l}}$ of

$$
T_{m}\left(u_{1}, \ldots, u_{l}\right)=(-1)^{\sum_{i<j} m_{i} m_{j}} \operatorname{det}\left[u_{i}^{\sigma_{m}(j)-1} T_{i}^{\left(a_{j}\right)}\left(u_{i}\right)\right]_{i, j=1, \ldots, l},
$$

where we use notation (5.20).

5.4. Corollary. Consider non-zero Laurent polynomials $A_{j}^{(a)}\left(u_{j}\right)$ in the definition of $T\left(u_{1}, \ldots, u_{l}\right)$ in (5.10). One can represent each of them in the form

$$
A_{j}^{(a)}(u)=u^{M_{j}^{(a)}} b_{j}^{(a)} \sum_{i \in \mathbb{Z}_{+}} a_{j, i}^{(a)} u^{i}, \quad 1 \leq j \leq l, 1 \leq a \leq s,
$$

for suitable choices of $M_{j}^{(a)} \in \mathbb{Z}, b_{j}^{(a)}, a_{j, i}^{(a)} \in \mathbb{C}, a_{j, 0}^{(a)}=1$, where all $b_{j}^{(a)}$ are non-zero. Then using analogous arguments as in Section 3.4 , one can deduce that there exist constants constants $c_{j, i}^{(a)} \in \mathbb{C}$, such that

$$
T_{j}^{(a)}(u)=u^{M_{j}^{(a)}} b_{j}^{(a)} \sum_{k=0}^{\infty} S_{k}\left(t_{1}^{(a)}+c_{j, 1}^{(a)}, t_{2}^{(a)}+c_{j, 2}^{(a)}, \ldots\right) u^{k} .
$$

Hence, $T_{j ; k}^{(a)}=b_{j}^{(a)} S_{k-M_{j}^{(a)}}\left(t_{1}^{(a)}+c_{i, 1}^{(a)}, t_{2}^{(a)}+c_{i, 2}^{(a)}, \ldots\right)$, and by (5.16) and Theorem 5.2, we obtain a polynomial tau-function of the $s$-component KP hierarch of charge $l \geq 0$, such that, in notation 
(5.20), it has the form

$$
\sum_{m \in \mathbb{Z}_{+}^{s},|m|=l}(-1)^{\sum_{i<j} m_{i} m_{j}} z^{m} \operatorname{det}\left[b_{i}^{\left(a_{j}\right)} S_{\alpha_{i}-M_{i}^{\left(a_{j}\right)}+1-\sigma_{m}(j)}\left(t_{1}^{\left(a_{j}\right)}+c_{i, 1}^{\left(a_{j}\right)}, t_{2}^{\left(a_{j}\right)}+c_{i, 2}^{\left(a_{j}\right)}, \ldots\right)\right]_{i, j=1, \ldots, l} .
$$

Remark 5.4. Note first, that the sum in (5.22) is finite, since there are only finitely many $m \in \mathbb{Z}_{+}^{s}$ such that $|m|=m_{1}+m_{2}+\cdots+m_{s}=l$. Note secondly, that any polynomial tau-function corresponds an element of charge 0 of the form

$$
\tau=g_{l} \wedge \cdots \wedge g_{1} \wedge e_{-l} \wedge e_{-l-1} \wedge e_{-l-2} \wedge \cdots,
$$

for certain $l$. This gives, when translating the charge by $l$ an element of the form (5.16). In this way we obtain that any polynomial tau-function of the $s$-component KP hierarchy is of the form (5.22), when translated over the lattice $\mathbb{Z}^{s}$ (see also Remark 5.2).

\section{Polynomial $\tau$-Functions of the Bilinear $\lambda$-KdV hierarchy}

In this section we consider the $\lambda$-KdV hierarchy for the partition $\lambda=\left(\lambda_{1}, \lambda_{2}, \ldots, \lambda_{s}\right)$ consisting of $s$ positive parts, which is a reduction of the $s$-component KP hierarchy introduced in [9]. We again use the ( $s$-component) boson-fermion correspondence.

6.1. Bilinear equations for the $\lambda$-KdV hierarchy. The $\lambda=\left(\lambda_{1}, \lambda_{2}, \ldots, \lambda_{s}\right)-\mathrm{KdV}$ hierarchy is a reduction of the $s$-component KP hierarchy, for which, in addition to equation (5.8), the tau-function satisfies the following equations

$$
\Omega_{j}(\tau \otimes \tau)=0, \quad j \in \mathbb{N}
$$

where

$$
\Omega_{j}=\sum_{a=1}^{s} \Omega_{j}^{(a)}, \quad \Omega_{j}^{(a)}=\sum_{k \in \mathbb{Z}+\frac{1}{2}} \psi_{k}^{+(a)} \otimes \psi_{j \lambda_{a}-k}^{-(a)} .
$$

6.2. Polynomial tau-functions for the $\lambda-\mathbf{K d V}$ hierarchy. See [12] for more details. For polynomial $s$-component tau-functions the additional equations (6.1) mean that

$$
\sum_{a=1}^{s} \frac{\partial \tau}{\partial t_{j \lambda_{a}}^{(a)}}=0, \quad j \in \mathbb{N}
$$

or, in the fermionic picture, that for an element (5.16), one has $\Lambda f_{j} \wedge \tau=0$, for $j=1, \ldots, l$, where the operator $\Lambda$ on $\mathbb{C}^{\infty}$ is defined by

$$
\Lambda e_{k}^{(a)}=e_{k-\lambda_{a}}^{(a)}, \quad 1 \leq a \leq s, k \in \mathbb{Z} .
$$

This means that (5.16) depends only on $r<|\lambda|$ elements $f_{j} \in \mathbb{C}^{\infty}$, namely that

$$
\tau=f_{1} \wedge \Lambda f_{1} \wedge \cdots \wedge \Lambda^{k_{1}-1} f_{1} \wedge f_{2} \wedge \cdots \wedge \Lambda^{k_{2}-1} f_{2} \wedge \cdots \wedge \Lambda^{k_{r}-1} f_{r} \wedge|0\rangle \text { and } \Lambda^{k_{j}} f_{j} \wedge|0\rangle=0,
$$

where $j=1, \ldots, r<|\lambda|$ and $k_{1}+k_{2}+\cdots+k_{r}=l$. Asuming that $f_{j}$ has the form

$$
f_{j}=\sum_{a=1}^{s} f_{j}^{(a)}, \quad f_{j}^{(a)}=\sum_{k=1}^{N_{j}^{(a)}} b_{k j}^{(a)} e_{k}^{(a)}, \quad j=1, \ldots, r, \quad a=1, \ldots, s,
$$

we obtain, since $\Lambda^{k_{j}} f_{j} \wedge|0\rangle=0$, that

$$
k_{j} \lambda_{a} \geq N_{j}^{(a)}, \quad \text { for all } 1 \leq j \leq r, 1 \leq a \leq s .
$$


Since we assume that $\Lambda^{k_{j}-1} f_{j} \wedge|0\rangle \neq 0$, we find that

$$
k_{j}=\max \left\{\left\lceil\frac{N_{j}^{(a)}}{\lambda_{a}}\right\rceil \mid 1 \leq a \leq s\right\}, \quad 1 \leq j \leq r .
$$

An element of the form (6.3) can be achieved by assuming that $\tau$ is the zero-mode of the Laurent series expansion of $T\left(u_{1}, \ldots, u_{l}\right)$, where $T\left(u_{1}, \ldots, u_{l}\right)$ depends only on $r s$ Laurent polynomials $B_{i}^{(a)}(u)$, with $1 \leq i \leq r$ and $1 \leq a \leq s$, such that $f_{j}$ can be replaced by the the zero-mode of

$$
\sum_{a=1}^{s} B_{i}^{(a)}(u) \sum_{\ell \in \mathbb{N}} \psi_{\frac{1}{2}-\ell}^{+(a)} u^{\ell}=\sum_{a=1}^{s} \sum_{k \in \mathbb{Z}} \sum_{\ell \in \mathbb{N}} B_{i, k}^{(a)} \psi_{\frac{1}{2}-\ell}^{+(a)} u^{k+\ell} .
$$

Hence, we can assume that $B_{i, k}^{(a)}=0$ for $k<-N_{j}^{(a)}$, in other words

$$
B_{i}^{(a)}(u)=u^{-N_{i}^{(a)}} b_{i}^{(a)} \sum_{\ell \in \mathbb{Z}_{+}} a_{i, \ell}^{(a)} u^{\ell} .
$$

Then the Laurent polynomials $A_{j}^{(a)}(u)$, for $1 \leq a \leq s$ and $1 \leq j \leq l$, which define $T\left(u_{1}, \ldots, u_{l}\right)$ by (5.10), (5.11), are given by

$$
\begin{aligned}
& A_{1}^{(a)}(u)=B_{1}^{(a)}(u), A_{2}^{(a)}(u)=u^{\lambda_{a}} B_{1}^{(a)}(u), \ldots, A_{k_{1}-1}^{(a)}(u)=u^{\left(k_{1}-1\right) \lambda_{a}} B_{1}^{(a)}(u), \\
& A_{k_{1}}^{(a)}(u)=B_{2}^{(a)}(u), \ldots, A_{k_{1}+k_{2}}^{(a)}(u)=u^{\left(k_{2}-1\right) \lambda_{a}} B_{2}^{(a)}(u), \\
& A_{k_{1}+k_{2}+1}^{(a)}(u)=B_{3}^{(a)}(u), \ldots \ldots, A_{l}^{(a)}(u)=u^{\left(k_{r}-1\right) \lambda_{a}} B_{r}^{(a)}(u),
\end{aligned}
$$

Recall the function $\sigma_{k}(x)$ for $k \in \mathbb{Z}^{r}$ on the interval $[0, l]$, introduced in Section 5.3. In addition, consider the step function $\gamma_{k}(x)$ on the interval $[1, l]$ which is piece-wise constant, continuous at all points except for points $\left\{k_{1}, k_{1}+k_{2}, \ldots, k_{1}+\cdots+k_{r-1}\right\}$, and

$$
\gamma_{k}(1)=1, \gamma_{k}\left(k_{1}+\cdots+k_{j}\right)=j \text {, for } j=1, \ldots, r .
$$

Then

$$
A_{j}^{(a)}(u)=u^{\left(\sigma_{k}(j)-1\right) \lambda_{a}} B_{\gamma_{k}(j)}^{(a)}(u) \text {, where the } B_{i}^{(a)}(u) \text { are as in (6.5). }
$$

Then (see also Remark 5.4 and 5.2) by formula (5.22), where we choose all $\alpha_{i}=0$ and $M_{i}^{(a)}=$ $\left(\sigma_{k}(i)-1\right) \lambda_{a}-N_{\gamma_{k}(i)}^{(a)}$, any polynomial tau-function of the $\lambda$-KdV hierarchy can be translated over the lattice $\mathbb{Z}^{s}$, such that it becomes a tau function of charge $l \geq 0$ for certain $l$ of the $\lambda$-KdV hierarchy of the form, in notation (5.20),

$$
\begin{aligned}
& \sum_{\substack{m \in \mathbb{Z}_{+}^{s},|m|=l}}(-1)^{\sum_{i<j} m_{i} m_{j}} z^{m} \\
& \quad \times \operatorname{det}\left[b_{\gamma_{k}(i)}^{\left(a_{j}\right)} S_{N_{\gamma_{k}(i)}^{\left(a_{j}\right)}-\left(\sigma_{k}(i)-1\right) \lambda_{a_{j}}+1-\sigma_{m}(j)}\left(t_{1}^{\left(a_{j}\right)}+c_{\gamma_{k}(i), 1}^{\left(a_{j}\right)}, t_{2}^{\left(a_{j}\right)}+c_{\gamma_{k}(i), 2}^{\left(a_{j}\right)}, \ldots\right)\right]_{i, j=1, \ldots, l},
\end{aligned}
$$

where the $k_{i}$ 's are given by (6.4).

\section{REFERENCES}

[1] E. Date, M. Jimbo, M. Kashiwara, T. Miwa, Operator Approach to the Kadomtsev-Petviashvili Equation. Transformation Groups for Soliton Equations III, J. Phys. Soc. Jpn. 50, (1981), no. 11, 3806-3812.

[2] E. Date, M. Jimbo, M. Kashiwara, T. Miwa, Transformation groups for soliton equations IV. A new hierarchy of soliton equations of KP type, Physica 4D (1982), 343-365.

[3] E. Date, M. Jimbo, M. Kashiwara, T. Miwa, Transformation groups for soliton equations, in: Nonlinear integrable systems - classical theory and quantum theory eds M. Jimbo and T. Miwa, World Scientific, (1983), 39-120. 
[4] F. Ingram, N. Jing, E. Stitzinger, Wreath product symmetric functions, Internat. J. Algebra 3 (2009), 1-19

[5] M. Jimbo, T. Miwa, Solitons and infinite-dimensional Lie algebras, Publ. Res. Inst. Math. Sci. 19 (1983), no. 3, $943-1001$.

[6] N. Jing, Vertex operators, symmetric functions and the spin group $\Gamma_{n}$, J. Algebra 138 (1991), 340-398.

[7] N. Jing, N. Rozhkovskaya, Generating functions for symmetric and shifted symmetric functions, arXiv:1610.03396, Journal of Combinatorics Volume 10, Number 1, 111-127, 2019.

[8] V. G. Kac, Vertex algebras for beginners. 2nd ed., University Lecture Series, 10. Amer. Math. Soc., Providence, RI, 1998.

[9] V. G. Kac, J. W. van de Leur, The $n$-component KP hierarchy and representation theory, in "Important developments in soliton theory", eds. A. S. Fokas, V. E. Zakharov, Springer Verlag (1993), 302-343; and in "Integrability, topological solitons and beyond", J. Math. Phys. 44 (2003), no. 8, 3245-3293.

[10] V. G. Kac, J. W. van de Leur, Equivalence of formulations of the MKP hierarchy and its polynomial tau-functions, Jpn. J. Math. 13 (2018), no. 2, 235-271.

[11] V. G. Kac, J. W. van de Leur, Polynomial tau-functions of BKP and DKP hierarchies, J. Math. Phys. 60 (2019), no. 7 .

[12] V. G. Kac, J. W. van de Leur, Polynomial tau-functions for the multi-component KP hierarchy, PRIMS (2020). arXiv:1901.07763.

[13] V. G. Kac, A. K. Raina, Bombay lectures on highest weight representations of infinite-dimensional Lie algebras, 1st ed., Advanced Series in Mathematical Physics, 2. World Scientific Publishing Co., NJ(1987).

V. G. Kac, A. K. Raina, N. Rozhkovskaya, Bombay lectures on highest weight representations of infinite dimensional Lie algebras, 2nd ed., World Scientific Publishing Co., NJ (2013).

[14] I. G. Macdonald, Symmetric functions and Hall polynomials, 2nd ed., Oxford Univ. Press, New York, 1995.

[15] N. Rozhkovskaya, Multiparameter Schur Q-functions are solutions of the BKP hierarchy, SIGMA Symmetry Integrability Geom. Methods Appl. 15 (2019), 065, 14 pages.

[16] M. Sato, Soliton equations as dynamical systems on infinite-dimensional Grassmann manifold, RIMS Kokyuroku, 439 (1981), 30-46.

[17] R. P. Stanley, Enumerative combinatorics. Vol. 2, Cambridge Univ. Press, Cambridge, (1999).

[18] Y. You, Polynomial solutions of the BKP hierarchy and projective representations of symmetric groups, in Infinite-dimensional Lie algebras and groups (Luminy-Marseille, 1988) Adv. Ser. Math. Phys. 7 (1989) 449-464.

[19] Y. You, DKP and MDKP hierarchy of soliton equations, Physica D 50 (1991), 429-462. USA

Department of Mathematics, Massachusetts Institute of Technology, Cambridge, Massachusetts 02139,

Email address: kac@math.mit.edu

Department of Mathematics, Kansas State University, Manhattan, KS 66502, USA

Email address: rozhkovs@math.ksu.edu

Mathematical Institute, Utrecht University, P.O. Box 80010, 3508 TA-Utrecht, The Netherlands

Email address: J.W.vandeLeur@uu.nl 